\title{
Characterisation of Silicon Carbide and Diamond Detectors for Neutron Applications $\ddagger$
}

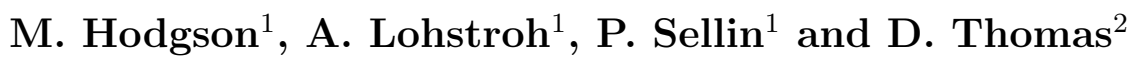 \\ ${ }^{1}$ Department of Physics, University of Surrey, Guildford, GU2 7XH, United Kingdom \\ ${ }^{2}$ NPL ${ }^{\odot}$, Teddington, TW11 0LW, United Kingdom \\ E-mail: michael.hodgson@becq.co.uk
}

\begin{abstract}
.
The presence of carbon atoms in silicon carbide and diamond makes the materials ideal candidates for direct fast neutron detectors. Furthermore the low atomic number, strong covalent bonds, high displacement energies, wide band gap and low intrinsic carrier concentrations make these semiconductor detectors potentially suitable for applications where rugged, high temperature, low gamma sensitivity detectors are required, such as Active Interrogation, Electronic Personal Neutron Dosimetry and Harsh Environment Detectors.

A thorough direct performance comparison of the detection capabilities of semiinsulating silicon carbide (SiC-SI), single crystal diamond (D-SC), polycrystalline diamond (D-PC) and a self-biased epitaxial silicon carbide (SiC-EP) detector has been conducted and benchmarked against a commercial silicon PIN (Si-PIN) diode, in a wide range of alpha (Am-241), beta ( $\mathrm{Sr} / \mathrm{Y}-90)$, ionising photon (65keV to $1332 \mathrm{keV}$ ) and neutron radiation fields (including $1.2 \mathrm{MeV}$ to $16.5 \mathrm{MeV}$ mono-energetic neutrons, as well as neutrons from AmBe and Cf-252 sources).

All detectors were shown to be able to directly detect and distinguish both the different radiation types and energies by using a simple energy threshold discrimination method. The $\mathrm{SiC}$ devices demonstrated the best neutron energy discrimination ratio $\left(E_{\max }[\mathrm{n}=5 \mathrm{MeV}] / E_{\max }[\mathrm{n}=1 \mathrm{MeV}] \approx 5\right)$, whereas a superior neutron/photon cross sensitivity ratio was observed in the D-PC detector $\left(E_{\max }[\mathrm{AmBe}] / E_{\max }[\mathrm{Co}-60] \approx 16\right)$. Further work also demonstrated that the cross sensitivity ratios can be improved through use of a simple proton-recoil conversion layer.

Stability issues were also observed in the D-SC, D-PC and SiC-SI detectors while under irradiation, that being a change of energy peak position and/or count rate with time (often referred to as polarisation effect). This phenomenon within the detectors was non-debilitating over the time period tested $(>5 \mathrm{~h})$ and as such, stable operation was possible.

Furthermore, the D-SC, self-biased SiC-EP and a semi-insulating SiC detector were shown to operate over the temperature range $-60^{\circ} \mathrm{C}$ to $+100^{\circ} \mathrm{C}$.
\end{abstract}

$\ddagger$ C)British Crown Owned Copyright 2017/AWE. 


\section{Introduction}

Silicon carbide (4H-SiC) and chemical vapour deposited diamond (CVD-D) semiconductors have been suggested as ideal devices for detecting neutrons in a number of specialist applications [1][2][3][4].

Despite a relatively small detection volume compared to other neutron detection techniques, they present the ability to directly detect fast neutrons due to the carbon atoms present, removing issues associated with conversion layers [5]. The thin material and low atomic number $(Z)$ also reduces the sensitivity to ionising photon radiation, which is essential as neutron fields are typically accompanied by a x-rays and/or gammarays.

The high threshold displacement energy of the material leads to a high level of radiation hardness in comparison to other common semiconductor detector materials [6][7], whereas the strong covalent bonds between atoms also make the materials mechanically strong.

Furthermore, the wide band gap and low intrinsic carrier concentration of these materials lead to low leakage current densities over a wide range of temperatures [6][8], allowing for stable operation in practical non-laboratory systems. This coupled with low capacitance values for bulk un-doped materials, potentially makes these detectors suitable for scaling up to large area devices without significant effects upon their capabilities.

In this work we present a complete and direct comparison of the main variants of $4 \mathrm{H}-\mathrm{SiC}$ and CVD-D to assess the suitability for neutron detection applications. In particular the neutron and gamma intrinsic efficiency as a function of radiation energy has been determined and compared to a standard silicon PIN photodiode (Si-PIN) to give a practical performance benchmark against a commercial semiconductor. Epitaxial $\mathrm{SiC}$ (SiC-EP), bulk semi-insulating $\mathrm{SiC}$ (SiC-SI), electronic grade single crystal D (D$\mathrm{SC}$ ) and polycrystalline D (D-PC) radiation detectors were all individually irradiated with alpha particles (Am-241), beta particles ( $\mathrm{Sr} / \mathrm{Y}-90)$, ionising photons $(0.065 \mathrm{MeV}$ to $1.332 \mathrm{MeV}, 3000 \mathrm{\gamma} / \mathrm{s}$ to $1 \times 10^{7} \gamma / \mathrm{s}$ ) and neutrons $(1.2 \mathrm{MeV}$ to $16.5 \mathrm{MeV}$, as well as AmBe and Cf-252, $250 \mathrm{n} / \mathrm{s}$ to $4500 \mathrm{n} / \mathrm{s})$. The SiC-EP, SiC-SI and D-SC detectors were also irradiated with $\mathrm{Am}-241$ alpha particles over a temperature range of $-60^{\circ} \mathrm{C}$ to $+100^{\circ} \mathrm{C}$.

\section{Neutron Applications}

\section{Active Interrogation}

Most active interrogation techniques utilise external radiation sources (usually neutrons and photons) to probe and identify objects under investigation. In principle this technique has been used for many years in medical X-ray machines, baggage scanners and in nuclear fuel cycle survey instruments [9]. However, since the mid-1990's there has also been significant interest in homeland security applications with the aim of 
preventing the illegal movement of nuclear material across ports and borders. This technique uses high fluence and energy radiation sources, usually accelerator based, to induce fission within the nuclear materials and detect the signature products [10].

The detection system used in security based active interrogation systems needs to be robust and capable of operating in relatively challenging radiological conditions. Regardless of the interrogation type, a mixed field of radiation will be present during operation from the initial interrogation pulse, fissile reaction, material activation and scattering. Therefore any suitable detection system must be able to distinguish between neutron and photon induced signals. Furthermore, in order to determine whether the characteristic neutron emissions are prompt fission neutrons (2-5 MeV) or delayed / scattered neutrons $(<2 \mathrm{MeV})[11]$, discrimination between different energies is also required [12].

The radiation sources are generally quick, high intensity pulses [10] with the subsequent characteristic fissile radiations ranging between prompt nanosecond emissions and delayed emissions over seconds, minutes and even hours. As such, the detector system must be able to operate over a wide dynamic range, as well as be able to either operate in or rapidly recover from, the high intensity interrogation pulse so that the induced emissions can be quickly detected ( $\sim \mathrm{ms}[12])$.

In order for practical roll-out at ports and borders the detectors must be stable during operation $(>1 \mathrm{~min})$ over a range of environmental conditions $\left(-30^{\circ} \mathrm{C}\right.$ to $\left.+55^{\circ} \mathrm{C}[12]\right)$. Furthermore regular exposures of high flux and energy radiation fields must not result in any radiation-induced damage over the operational life-time of the detection system (typically around 5 years as a minimum [12]).

\section{Electronic Personal Neutron Dosimetry}

Electronic personal neutron dosemeters are an established international industry spanning nuclear power, medical, defence and emergency services. The dominant models on the market use multiple silicon photodiodes with various filters or convertor layers to detect beta, X-ray, gamma and/or neutron radiation [13][14][15].

Neutron detection in electronic dosemeters for most devices is achieved by using Si detectors with several neutron conversion layers [14][15] to give a characteristic energy response, which in the most part, is similar for the majority of devices on the market [16][17]. However, maintaining a flat dose equivalent response over such a wide range of energies $(0.025 \mathrm{eV}$ to $15 \mathrm{MeV})$ is extremely difficult, with the neutron personal dose equivalent relative response of these devices often ranging from $\approx 0.1$ to 15 [16].

One of the reasons for this non-uniformity is related to how the different detector channels are combined. Detection thresholds within these devices are set in-order to minimise the cross-sensitivity to gamma radiation on the same channel. However, this threshold will also limit the detection of lower energy conversion particles, effecting the overlap between the detector channels and subsequently how they are combined.

A further issue with these devices is that the fundamental sensitivity to neutrons 
(counts/dose) is usually quite poor, requiring relatively high doses before statistically significant readings can be considered. Although this is in some way a result of the inefficiencies related to conversion layers [5], it is more likely related to the relatively small size of the detectors limiting the probability of detection. Although larger area detectors allow more particles to be detected, increasing the ability to detect fast neutrons, this is at the expense of higher leakage current, capacitance and an increase in gamma cross-sensitivity (i.e. more gamma-ray photons are detected).

\section{Harsh Environment}

The global drive for continual data analysis of components and processes is leading the need for increasingly challenging detector requirements. One of the most difficult areas to address is for harsh environment applications where sensitive detectors need to operate over wide temperature ranges, high pressures, large radiation fields or even under high mechanical stress.

Applications in nuclear industry are often driven by temperatures up to several hundred degrees Celsius [18] and integrated neutron exposures up to $10^{18}$ neutrons per $\mathrm{cm}^{2}[19][20][21]$. Significant ionising photon radiation is also present, requiring both neutron-gamma discrimination and ionising photon radiation hardness.

Within spacecraft and aerospace, integrated radiation doses are high due to solar flares and planetary magnetospheric radiation $(>0.2 \mathrm{mSv} /$ day ambient dose equivalent [22][23]), whereas mechanical stresses may be extensive during transit [3]. Detectors for automobile applications, particularly in engines, not only have to survive significant mechanical stress but also temperatures potentially up to $1000^{\circ} \mathrm{C}$ [24].

In natural resource well logging (oil and gas) the detection systems operate in environments up to $\approx 300^{\circ} \mathrm{C}[4]$, pressures $>1000$ atm and relatively large radiation doses due to the active interrogation method and background radiation from the rock. The detectors must also be able function after extreme mechanical stress when installed in drilling heads [25].

\section{Experimental Methods}

\section{Sample Preparation}

A summary of detectors under investigation has been given in Table 1. The D-SC detector was fabricated from Element Six Ltd ${ }^{\circledR}$ electronic grade, chemical vapour deposition (CVD) diamond with $4 \times 4 \mathrm{~mm}^{2}$ platinum contacts [26][27]. The D-PC detector was fabricated from Diamond Detectors Limited ${ }^{\complement}$ CVD diamond material with $\varnothing 6.5 \mathrm{~mm}$ gold contacts [27][28].

The SiC-EP detector was fabricated from Cree ${ }^{(0)}$ material [29][30] consisting of an $50 \mu \mathrm{m}$ epitaxial layer of n-type $4 \mathrm{H}-\mathrm{SiC}\left(5 \times 10^{14} \mathrm{~cm}^{-3}\right)$ grown on $360 \mu \mathrm{m}$ of $4 \mathrm{H}-\mathrm{SiC}$ bulk semi-insulating substrate. Ti/Pt/Au Schottky contacts were applied and annealed to $1000^{\circ} \mathrm{C}$, where as $\mathrm{Ni} / \mathrm{Au}$ was used for the ohmic contacts. 


\begin{tabular}{|c|c|c|c|c|c|c|}
\hline Detector & $\begin{array}{l}\text { Material } \\
\text { Manufacturer }\end{array}$ & Material & $\begin{array}{c}\text { Thickness } \\
(\mu \mathrm{m})\end{array}$ & Contact Material & $\begin{array}{l}\text { Contact } \\
\text { Size } \\
(\mathrm{mm}) \\
\end{array}$ & $\begin{array}{l}\text { Contact } \\
\text { Thickness } \\
(\mathrm{nm}) \\
\end{array}$ \\
\hline Si-PIN & Hamamatsu@ & $\mathrm{Si}$ & $\approx 300$ & - & $3.6 \times 3.6$ & - \\
\hline SiC-EP & $\mathrm{Cree} \bigodot$ & $4 \mathrm{H}-\mathrm{SiC}$ & $50+360$ & (O) $\mathrm{Au} / \mathrm{Pt} / \mathrm{Ti}: \mathrm{Ni} / \mathrm{Au}(\mathrm{S})$ & $\varnothing 5$ & $150 / 30 / 20: 100 / 100$ \\
\hline SiC-SI & $\mathrm{Cree}^{(\mathrm{C})}$ & $4 \mathrm{H}-\mathrm{SiC}$ & 350 & (O) $\mathrm{Au} / \mathrm{Ni}: \mathrm{Ti} / \mathrm{Pt} / \mathrm{Au}(\mathrm{S})$ & $5 \times 5$ & $1500 / 40: 60 / 40 / 2000$ \\
\hline SiC-SI-Cr3 & $\mathrm{Cree} @$ & $4 \mathrm{H}-\mathrm{SiC}$ & 350 & (O) $\mathrm{Au} / \mathrm{Ni}: \mathrm{Ti} / \mathrm{Pt} / \mathrm{Au}(\mathrm{S})$ & $9 \times 9$ & $100 / 100: 30 / 20 / 100$ \\
\hline $\mathrm{SiC}-\mathrm{SI}-\mathrm{Cr} 4$ & Cree $\left.{ }^{(}\right)$ & $4 \mathrm{H}-\mathrm{SiC}$ & 350 & (O) $\mathrm{Au} / \mathrm{Ni}: \mathrm{Ti} / \mathrm{Pt} / \mathrm{Au}(\mathrm{S})$ & $2 \times 2$ & $1500 / 40: 30 / 20 / 100$ \\
\hline SiC-SI-Cr5 & Cree $\left.{ }^{(}\right)$ & $4 \mathrm{H}-\mathrm{SiC}$ & 350 & (O) $\mathrm{Au} / \mathrm{Ni}: \mathrm{Ti} / \mathrm{Pt} / \mathrm{Au}(\mathrm{S})$ & $5 \times 5$ & $1500 / 40: 30 / 20 / 100$ \\
\hline D-SC & Element Six $\subset$ & CVD-D & 500 & $\mathrm{Pt}: \mathrm{Pt}$ & $4 \times 4$ & $120: 120$ \\
\hline $\mathrm{D}-\mathrm{PC}$ & Diamond Detectors Ltd ${ }^{\circledR}$ & CVD-D & 300 & $\mathrm{Au}: \mathrm{Au}$ & $\varnothing 6.5$ & $100: 100$ \\
\hline
\end{tabular}

Table 1. Main detectors under investigation. The values given in the format X:X correspond to the position relative to the detector material, in this case represented by ' $\because$. Furthermore, in the 'Contact Material' column (S) and (O) correspond to Schottky barrier contact and ohmic contact respectively.

As a significant amount of work has demonstrated the radiation detection capabilities of epitaxial silicon carbide [31][32][33][34] it was decided that the selfbiased (applied 0V) radiation detection performance of the SiC-EP detector would be determined. This mode of operation is a particularly interesting concept for the applications discussed within this paper, as not only does it allow for reduced electronics and power consumption, the low bias results in smaller depletion regions () which could decrease the active volume, but also potentially minimise gamma cross-sensitivity in the detectors [35], as shown by Equation 1 [36],

$$
x_{d e t} \cong \sqrt{2 \epsilon \epsilon_{0} V \mu \rho_{e}}
$$

where $\epsilon$ is the dielectric constant of the semiconductor material, $\epsilon_{0}$ is the permittivity of free space, $V$ is the bias, $\mu$ is the mobility of the majority charge carrier and $\rho_{e}$ is the detector resistivity.

The semi-insulating $\mathrm{SiC}$ detector (SiC-SI) was fabricated from Cree ${ }^{\complement} 4 \mathrm{H}-\mathrm{SiC}$ material with $5 \times 5 \mathrm{~mm}^{2} \mathrm{Ti} / \mathrm{Pt} / \mathrm{Au}$ Schottky contacts and $\mathrm{Ni} / \mathrm{Au}$ ohmic contacts [33]. Additional SiC-SI detectors were fabricated from $350 \mu \mathrm{m} 4 \mathrm{H}-\mathrm{SiC}$ (on-axis) Cree ${ }^{\circledR}$ material with $\mathrm{Ti} / \mathrm{Pt} / \mathrm{Au}$ Schottky contacts and $\mathrm{Ni} / \mathrm{Au}$ ohmic contacts were sputter deposited onto the material over areas covering $9 \times 9 \mathrm{~mm}^{2}(\mathrm{Cr} 3), 2 \times 2 \mathrm{~mm}^{2}$ (Cr4) and $5 \times 5 \mathrm{~mm}^{2}$ (Cr5). Each sample was cleaned with isopropanol (heated to $85^{\circ} \mathrm{C}$ ), methanol (heated to $85^{\circ} \mathrm{C}$ ) and acetone (heated to $50^{\circ} \mathrm{C}$ in an ultrasonic bath) prior to sputtering. For the $\mathrm{Ni} / \mathrm{Au}$ ohmic contacts the samples were annealed to $150^{\circ} \mathrm{C}$ for $60 \mathrm{~min}$ and $980^{\circ} \mathrm{C}$ for $2 \mathrm{~min}$ in a MTI GSL-1100X tube furnace $\left(10^{\circ} \mathrm{C} / \mathrm{min}\right.$ heat $/$ cool profile $)$ with a constant flow of nitrogen gas. Similarly the Ti/Pt/Au Schottky contacts were annealed to $500^{\circ} \mathrm{C}$ for 30min using the same technique.

A commercial Hamamatsu ${ }^{\circledR}$ silicon PIN photodiode (Si-PIN), type S1223-01, was used as a comparison benchmark. The standard package window was carefully removed in order that the detector could be utilised for alpha and beta detection, as demonstrated by Gooda and Gilboy [37]. 


\section{Dark Leakage Current}

The current-voltage (IV) relationship of each detector was determined using a combined Keithley 487 Picoammeter and Voltage Supply in series with a $10 \mathrm{k} \Omega$ resistor. Each test was conducted within a light sealed diecast metal test box at room temperature $(\approx 22$ ${ }^{\circ} \mathrm{C}$ ), with each voltage increment given up to 100s to stabilise before being read.

Between $0 \mathrm{~V}$ and the $\pm 5 \mathrm{~V}$ region the current was measured for $\pm 0.1 \mathrm{~V}$ increments so as to give the best representation of the bulk resistivity of the material. At higher biases the surface impurities and the contact materials dictate the resistivity measured as a result of variations in the space charge region across an interfacial layer between the semiconductor and the metal contact [38][39]. The work of Bolotnikov et al. has shown that this region subtends $\pm 1 \mathrm{~V}$ in CZT semiconductor detectors [38][39], but the concept can be applied to other high resistivity materials as well.

From the straight-line fit of the current-voltage relationship, the gradient relates to the resistance $(R)$ and the resistivity $\left(\rho_{e}\right)$ of a semiconductor detector of thickness $x$ can be given by,

$$
\rho_{e}=\frac{a}{x}
$$

where $a$ is the area of the contact. This equation assumes that the leakage current is $0 \mathrm{~A}$ at $0 \mathrm{~V}$ bias and that the detector is a simple parallel electrode detector. In reality this is not always the case when measuring fully fabricated detectors (rather than just the material), due to variations caused by fabrication and slight offsets in the measuring equipment at the very low currents measured. However, even in these circumstances Equation 2 still operates as a good and simple figure-of-merit check for the resistivity of the material.

\section{Radiation Spectroscopy}

For all radiation based testing the detectors were mounted within a light sealed diecast metal test box and connected to ORTEC 142A charge sensitive preamplifiers; ORTEC 570 or 572 shaping amplifiers; an ORTEC 710 quad-bias supply; and ORTEC EasyMCA multi-channel analyser with associated Maestro software. Energy calibration of the detectors was conducted using pulser-capacitor calibrations as described by Siegbahn [40],

$$
\begin{array}{rlll}
E_{\text {pulser }}= & V_{\text {pulser }} \times & W \\
(k e V) & (m V) & (\mathrm{eV}) & C_{\text {cap }} \\
& (p F)
\end{array}
$$

Where $E_{\text {pulser }}$ is the equivalent energy deposited into the electronics system, $V_{\text {pulser }}$ is the amplitude of the input pulser peak, $W$ is the average electron-hole creation energy for a given material, and $C_{c a p}$ is the capacitance value of a capacitor connect in series with a preamplifier and pulser. By plotting $E_{\text {pulser }}$ against the resultant peak centroid position on the MCA output, a linear relationship can be observed, the equation of which gives the calibration factor for the detection system. 
The D-SC, D-PC and SiC-SI detectors have all demonstrated some form of the so-called polarisation effect during irradiation, that being a change in the acquired spectrum and / or count rate with time [27][41][42][43]. This particular effect is prevalent in low doped, wide band gap semiconductors (D [44], SiC [45], CdZnTe [46] and CdTe [47]) and is a result of charge carriers being trapped for long periods of time, leading to a change in the space charge distribution [36]. As such, between each radiation exposure the detectors were exposed to at least 15 minutes of room ambient light, while at $0 \mathrm{~V}$ bias, in order to de-polarise the detector. This procedure was found to reset the peak position, count rate and polarisation rate performance of the detectors with a reasonable level repeatability.

For the applications discussed, the detector must be able to detect neutrons in a mixed field environment. Most neutron sources result in ionising photon and neutrons fields, but reactions with the surrounding environment mean that charged particles are also likely to be present in a high energy neutron field and should be considered.

In order to detect neutrons within this environment the detector should be either insensitive to the interference radiation or be able to discriminate against it. As such, the detectors were tested over the standard range of radiation types (alpha, beta, X-ray, gamma, neutron) in order to characterise their susceptibility.

All alpha spectra tests were conducted with a $3.7 \mathrm{kBq}$ Am-241 source $(5.485 \mathrm{MeV})$ inside a vacuum chamber at a pressure of $8 \times 10^{-2}$ mbar. Alpha polarisation testing was conducted in air with a $60 \mathrm{kBq}$ Am-241 source (actual emission at $3.976 \mathrm{MeV}$ ). Similarly beta irradiations were conducted in air with $178 \mathrm{kBq}$ Sr-90/Y-90 source $(0.544 \mathrm{MeV} / 2.270 \mathrm{MeV})$.

$\mathrm{X}$-ray and gamma ray irradiations were conducted at AWE using either a Pantak Xray generator with ISO 4037 Narrow Spectrum filtration [48] or a gamma irradiator with Cs-137 (0.6617MeV) or Co-60 (1.1732MeV, 1.3325MeV) sources of various activities. Unless otherwise stated the ionising photon ambient dose equivalent rates were $\approx 6 \mathrm{mSv} / \mathrm{h}$.

Mono-energetic neutron exposures were conducted at the National Physical Laboratory ${ }^{\circledR}$ (NPL). Radionuclide neutron exposures (Cf-252, AmBe) were conducted at NPL ${ }^{\circledR}$, AWE and Thermo Fisher Scientific ${ }^{\complement}$ (Beenham). Where possible these tests were conducted in ambient dose equivalent fields greater then $6 \mathrm{mSv} / \mathrm{h}$ and irradiated for times exceeding 2 hours in order to obtain reasonable counting statistics in all channels. For mono-energetic $16.5 \mathrm{MeV}$ neutrons the data presented uses the standard calibration setting and as such the end point energy is reduced.

Before every irradiation the detectors were exposed to at least 15 minutes of room ambient light, while at $0 \mathrm{~V}$ bias, in order to remove any polarisation within the detector.

\section{Fast Neutron Convertor Layers}

The most statistically probable interaction method for fast neutrons is elastic recoil scattering where the incident neutron transfers a portion of its kinetic energy to the 
absorbing material through direct collisions.

Elastic interactions result in a change of direction and energy for the incident neutron as well as a gain of energy and momentum for the nucleus which recoils. If the energy transferred to this nucleus is such that its velocity is greater than that of its orbital electrons, it will lose those electrons and move through the medium as a heavy charged particle.

As discussed by Knoll [36], the maximum transfer of energy between the incident neutron and target nucleus occurs when the scattering angle of the incident neutron is $180^{\circ}$ or the recoil angle of the nucleus is $0^{\circ}$. However the energy transfer is fundamentally limited by the atomic mass of the absorber nuclei $(A)$, as given by Equation 4 [36],

$$
E_{r}=\frac{4 A}{(1+A)^{2}}\left(\cos ^{2} \theta\right) E_{n}
$$

where $E_{r}$ and $\theta$ are the recoil energy and angle of the nucleus respectively.

For carbon and silicon the maximum relative energy conversion ratio $\left(E_{r} / E_{n}\right)$ of the incident neutron $\left(E_{n}\right)$ and the nucleus are 0.28 and 0.13 respectively, allowing for potential use as a direct neutron detectors [27].

However, for hydrogen the limit is 1.0 , due to its similar mass relative to a neutron, making it theoretically possible for complete neutron energy transfer following collisions. For this reason hydrogenous materials such as water or plastic are by far the most popular mediums for neutron shielding and moderation. Hydrogenous materials can also be used for direct detection (such as proton recoil detectors) or for conversion detection, where the recoil hydrogen atom (proton) is detected by a charge particle detector [33][49][50][51][52].

Subject to the fast neutron energies, the potential energy of these conversion protons is high and as such it is possible the penetration depth will be more than the thickness of the detector (a $16.5 \mathrm{MeV}$ proton could travel $\approx 1.7 \mathrm{~mm}$ in $\mathrm{Si}$ or $\mathrm{C}[53]$ ). Similarly if the convertor layer is too thick, the conversion protons may not escape and fast neutrons will be attenuated. Furthermore, it is also possible that the conversion protons will be emitted at non-incident angles; depositing a fraction of their energy or not even hitting the detector. Consequently, the addition of any form of conversion layer will result in a maximum intrinsic efficiency limited by the sequence of probabilities for interaction and detection [5].

Within this investigation a $25 \mu \mathrm{m}$ thick piece of Kapton was used as a hydrogenous convertor layer to study efficiency and the operational stability of this detection modality. The distance from convertor layer to the detector varied from detector-todetector due to the arc of the bonding wire (the two could not touch due to the Kapton adhesive) but it was generally around 1-2mm.

\section{Temperature Testing}

Temperature testing was limited to detectors mounted on small ceramic boards and conducted in a vacuum cryostat from $-60^{\circ} \mathrm{C}$ and $+100^{\circ} \mathrm{C}(213 \mathrm{~K}$ to $373 \mathrm{~K})$ with the 
$60 \mathrm{kBq}$ Am-241 source. The boards with detectors were secured on the Heat / Cool plate within the cryostat using thermal coupling paste and a physical latch. A thermistor was also placed within the cryostat such that it was in contact with the ceramic board and therefore acted as a direct check of the temperature of the detector during testing. The preamp, shaping amp, bias supply and MCA were all outside the vacuum cryostat and not subject to the temperature variations.

Due to the mounting of the SiC-SI and Si-PIN detectors (PCB), as well as the size of the D-PC detector, this testing was limited to the D-SC and SiC-EP detectors, as well as the SiC-SI-Cr5 sample, which was deemed a suitable substitution for the SiC-SI detector.

The cryostat was pumped down to a vacuum of $6.4 \times 10^{-1}$ mbar for all tests conducted. The Heat / Cool plate was connected to either an Oxford Instrument ITC 503 or Mercury ITC, subject to the requirement of cooling or heating respectively, as described in [4]. For cooling a supply of liquid nitrogen was pumped throughout the system, with the temperature automatically regulated by the ITC 503 instrument.

\section{Results}

\section{Dark Leakage Current}

The current-voltage characteristics of the detectors are shown in Figure 1, as well as for the three samples of Cree semi-insulating material with different size contacts (SiCSI-Cr3, SiC-SI-Cr4, SiC-SI-Cr5). In addition to this, the resistivities of the diamond detectors and SiC-SI are given in Table 2. The resistivities of the SiC-EP and Si-PIN detectors were not determined as the more complex doping and contact arrangement did not reflect the simplified geometry required for Equation 2.

All the detectors under test showed very good leakage currents over the ranges tested, in the order of $\mathrm{nA}$, which would suggest good operation for most radiation detection applications [36], in particular particle detection and electronic personal dosimetry.

As would be expected for a wide band gap, low intrinsic carrier concentration detector, the diamond samples demonstrated the lowest leakage current over the range of biases tested. The D-PC detector provided a lower and more stable leakage current compared to the D-SC, which is a reasonable observation when considering the calculated resistivities of the two materials $2 \pm 1 \times 10^{15} \Omega \cdot \mathrm{cm}$ compared to $7.1 \pm 0.2 \times$ $10^{12} \Omega \cdot \mathrm{cm}$ respectively. It should be noted that the larger error in the D-PC value is a result of the observed low leakage currents over the tested range $( \pm 5 \mathrm{~V})$ being at the limits of detectability of the picoammeter, in the order of fA.

The doped detectors (SiC-EP, Si-PIN) had higher leakage currents relative to the other detectors, as would be expected for the intentional increase in carrier concentration due to doping.

Comparisons of the three semi-insulating $\mathrm{SiC}$ Cree detectors (SiC-SI-Cr3, SiC-SI- 
Cr4, SiC-SI-Cr5) show that the leakage currents are in a similar order of magnitude, particularly at high operating voltages, despite the different contact areas.

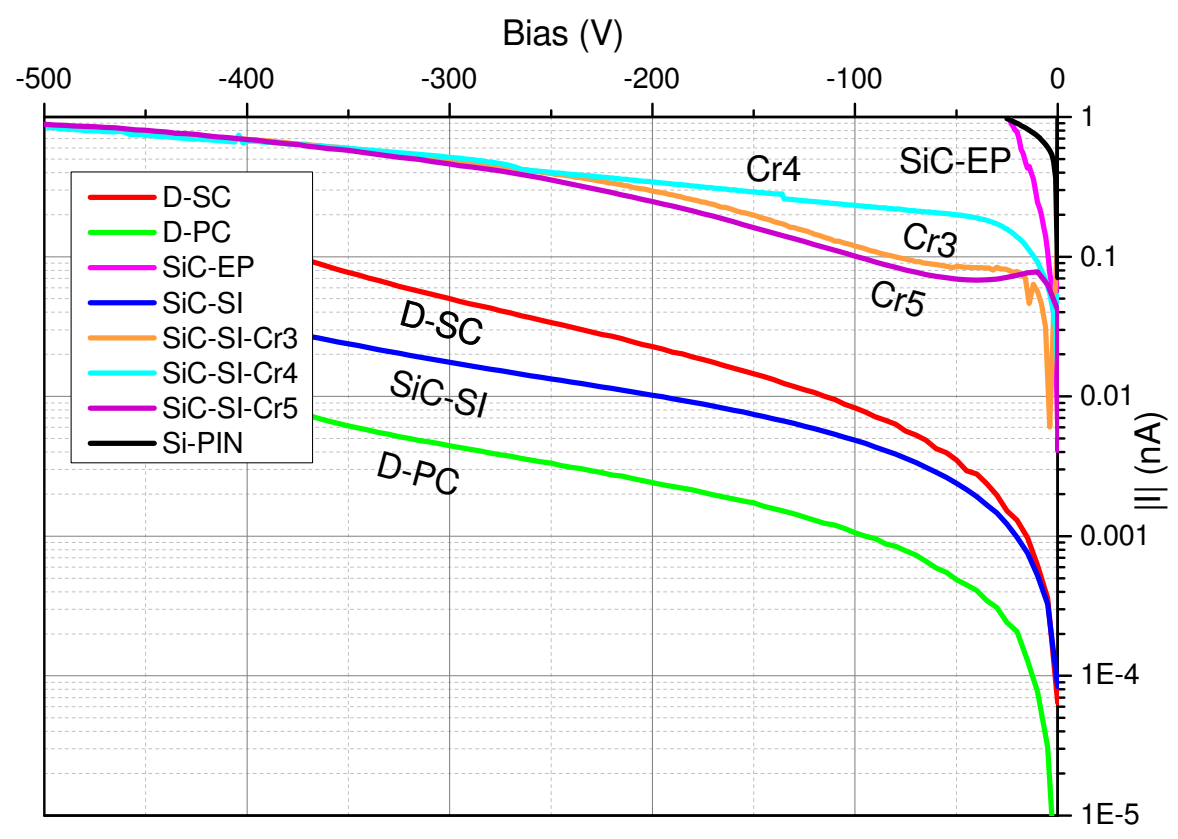

Figure 1. Current magnitude against voltage, normalised to the $0 \mathrm{~V}$ inflection point. The settling time following bias change was 1s, with 10 samples taken at each bias. Detectors SiC-SI-Cr3, SiC-SI-Cr4 and SiC-SI-Cr5 are Cree semi-insulating SiC detectors with contact sizes $9 \times 9 \mathrm{~mm}, 2 \times 2 \mathrm{~mm}$ and $5 \times 5 \mathrm{~mm}$ respectively.

For Active Interrogation and Electronic Personal Neutron Dosimtery applications, these observations are of particular interest as it suggests that low noise, larger area detectors are possible. These results also validate the fabrication technique used (i.e. little variation from detector-to-detector), although comparison between SiC-SI and SiC-SI-Cr5 suggests that the fabrication technique for the SiC-SI-Cr detectors could be improved further. Both these detectors were made from Cree material with similar resistivities and had the same contact area, but at higher biases the SiC-SI-Cr detector leakage currents are an order of magnitude greater.

The measured resistivity of the D-PC samples was similar to that of published values by Schirru et al. $\left.\left(1.4 \times 10^{15} \Omega \mathrm{cm}\right)[28]\right)$ when considering the uncertainties.

The resistance at a given bias $(+220 \mathrm{~V})$ for the D-SC sample was $0.76 \pm 0.06 \times 10^{14} \Omega$ compared to $2.2 \times 10^{14} \Omega$ as published by Abdel-Rahman et al. [26], however as no guard ring was utilised in these detectors, leakage currents may have been influenced by surface effects.

The leakage current of the SiC-EP detector was similar to that reported by Bruzzi et al. in the RD50 collaboration [29] ( $<10 \mathrm{nA}$ at $-100 \mathrm{~V})$. The Si-PIN also performed as per the manufacture's expected leakage current response $(\approx 0.1 \mathrm{nA}$ at $-10 \mathrm{~V})$. 


\begin{tabular}{|c|c|c|c|}
\hline Detector & $\begin{array}{c}\text { Typical } \\
\text { Operating } \\
\text { Bias } \\
(\mathrm{V})\end{array}$ & $\begin{array}{c}\text { Leakage } \\
\text { Current } \\
\text { Density } \\
\left(\mathrm{nA} / \mathrm{cm}^{2}\right)\end{array}$ & $\begin{array}{c}\text { Resistivity } \\
(\Omega \cdot \mathrm{cm})\end{array}$ \\
\hline $\mathrm{Si}-\mathrm{PIN}$ & -25 & $-8.0 \pm 0.1$ & - \\
\hline $\mathrm{SiC}-\mathrm{EP}$ & 0 & $-6560 \pm 1$ & - \\
\hline $\mathrm{SiC}-\mathrm{SI}$ & -400 & $-0.08 \pm 0.02$ & $0.53 \pm 0.01 \times 10^{13}$ \\
\hline SiC-SI-Cr3 & -400 & $-0.8 \pm 0.1$ & $5.8 \pm 0.1 \times 10^{13}$ \\
\hline SiC-SI-Cr4 & -400 & $-4.2 \pm 0.1$ & $12 \pm 2 \times 10^{13}$ \\
\hline SiC-SI-Cr5 & -400 & $-2.7 \pm 0.1$ & $0.44 \pm 0.01 \times 10^{13}$ \\
\hline $\mathrm{D}-\mathrm{SC}$ & -200 & $-0.077 \pm 0.009$ & $7.1 \pm 0.2 \times 10^{12}$ \\
\hline $\mathrm{D}-\mathrm{PC}$ & -400 & $-0.011 \pm 0.003$ & $2 \pm 1 \times 10^{15}$ \\
\hline
\end{tabular}

Table 2. Key performance criteria of the detectors under test. The leakage current density was taken at the typical operating bias. Operational characteristics of D-SC, D-PC and SiC-SI have been demonstrated in [27].

\section{Radiation Spectroscopy}

Figure 2 shows the spectral response of each detector to multiple radiation types. Each spectrum has been normalised to the incident radiation flux. This type of normalisation gives an indication of the relative sensitivities for each radiation type. For example, the vertical axis position of the alpha radiation data in the graphs is higher than other radiation types, representing more counts per incident radiation particle. This is reasonable considering the large energy and shallow penetration depth of alpha particles, meaning all of the incident particle's energy will be transferred to the detector.

The incident flux of the alpha sources were determined through the finite source approximation described by Knoll [36] and Ruby [54], with consideration given for selfattenuation losses. For X-ray and Gamma-ray exposures the incident flux calculation utilised the calibrated dose rate from the test facilities and conversion factors taken the ISO4037 series [48]. The incident flux of the neutron sources was calculated by the test house, where applicable.

Normalisation to the incident flux was not possible for the beta radiation source as insufficient information was available on the surface emission rate and the tests were conducted in air due to safety pressure restrictions on the source. However, the data presented in Figure 3 has been normalised to the contact surface area. As the detectorto-source distance was similar for most detectors, this normalisation is proportional to the incident flux when comparing detectors.

A similar issue occurs when considering neutron conversion layers, as the true number of particles incident upon the detector includes scattered recoil particles and neutrons, as well as un-scattered neutrons. Consequently the data has been plotted separately in Figure 4 for D-SC and the intrinsic detection efficiency (total counts / total incident particles) shown in Table 3.

The results presented in Figure 2 clearly show that all the detectors are capable 


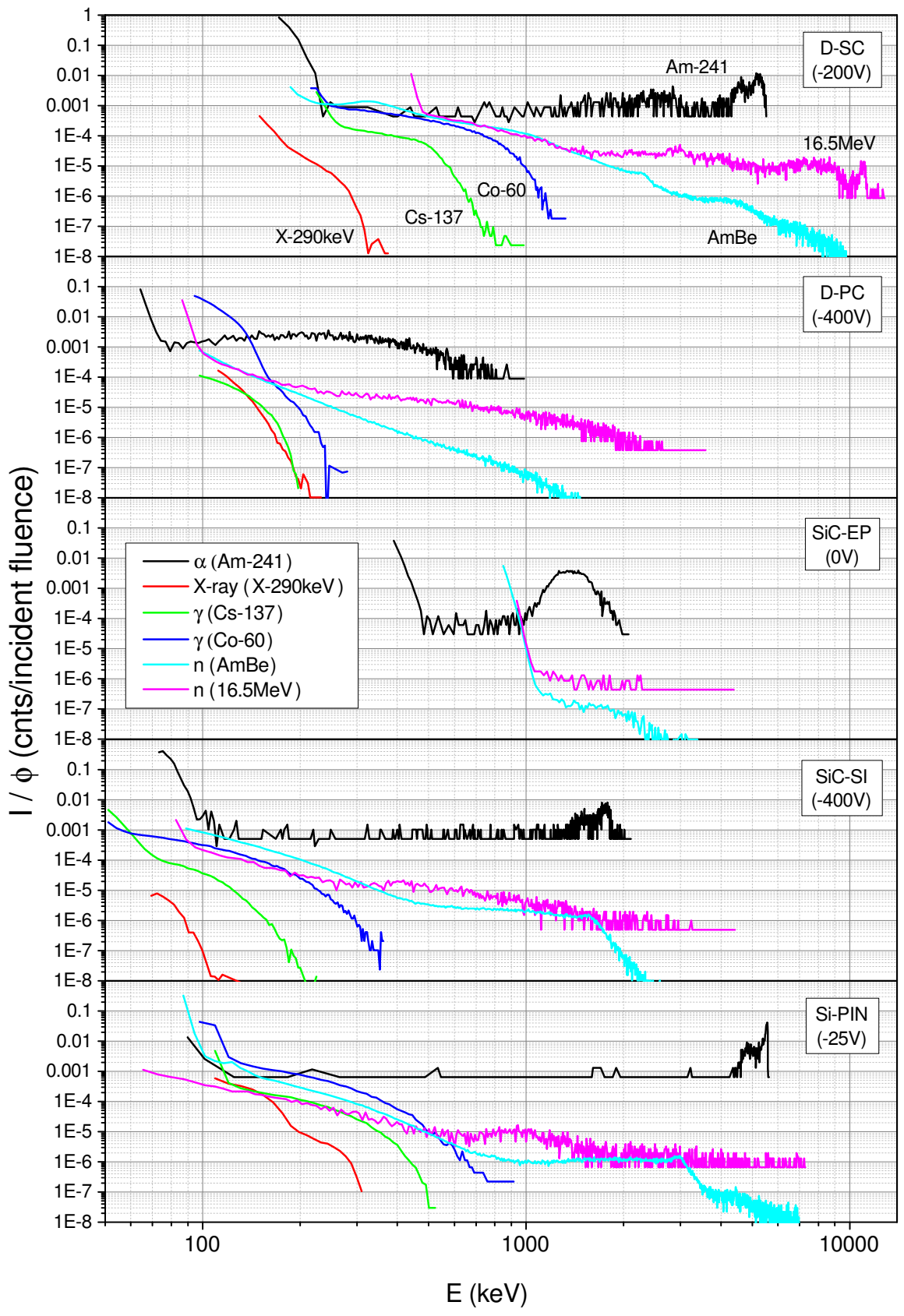

Figure 2. Count rate $(I)$, normalised to incident flux $(\phi)$, against energy $(E)$ as a function of alpha, X-ray, gamma, radionuclide neutron and mono-energetic neutron sources. Non-alpha data were taken at $\geq 6 \mathrm{mSv} / \mathrm{h}$. The perceived "levels" in some spectra (particularly alpha) are artefacts of the flux normalisation and are associated with the detector's ability to detect that radiation type. The calibration for the $16.5 \mathrm{MeV}$ neutrons was optimised for low energies, resulting in high level threshold at $<16.5 \mathrm{MeV}$ for the detectors under test. 


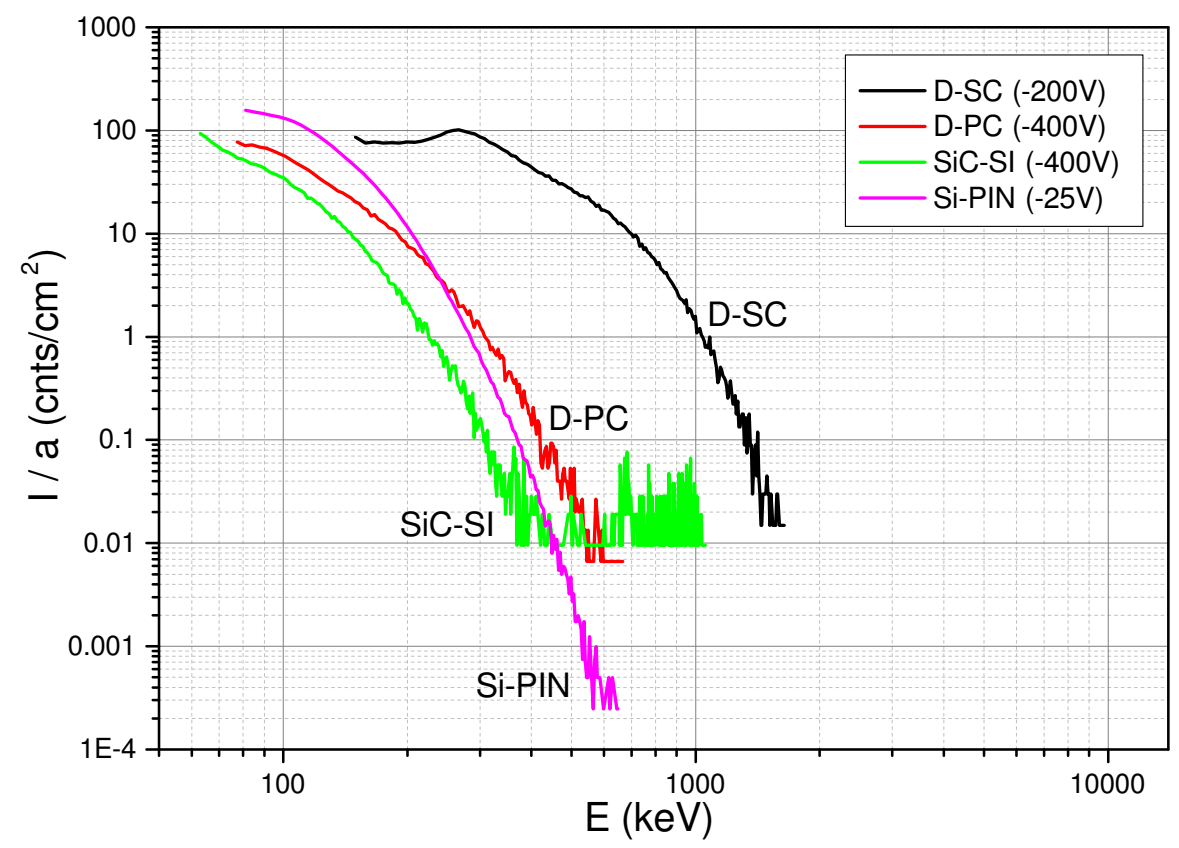

Figure 3. Count rate $(I)$, normalised to contact surface area $(a)$, against energy $(E)$ for a ${ }^{90} \mathrm{Sr} /{ }^{90} \mathrm{Y}$ beta source. All spectra were taken in air.

of discriminating between each of the radiation types and energies tested using a simple energy threshold / discriminator method. This is particularly important for active interrogation applications when comparing the Co-60 gamma and AmBe neutron spectra, as the average energies for these two sources $(1.2 \mathrm{MeV}$ and $4.1 \mathrm{MeV})$ are similar to those found following fission [55].

When considering charged particles (alpha and beta) the region for direct neutron detection above the threshold level reduces for SiC-EP, SiC-SI and Si-PIN. For D-SC and D-PC, the neutron detection capabilities above the alpha and beta thresholds remain very good.

There is little difference between the neutron detection capabilities of the Si-PIN and SiC-SI detectors, although as presented in Table 4, comparison of the different neutron end point energies $\left(E_{\max }\right)$ showed that both $\mathrm{SiC}$ detectors had very good neutron energy discrimination ability $\left(E_{\max }[\mathrm{n}=5 \mathrm{MeV}] / E_{\max }[\mathrm{n}=1 \mathrm{MeV}] \approx 5\right)$. For Si-PIN discrimination was still possible, but to a lower extent $\left(E_{\max }[\mathrm{n}=5 \mathrm{MeV}] /\right.$ $\left.E_{\text {max }}[\mathrm{n}=1 \mathrm{MeV}] \approx 2\right)$.

Despite demonstrating the highest intrinsic neutron efficiencies (Table 3), highlighting the advantage of direct carbon-neutron recoil reactions, both the D-SC and D-PC gave a lower neutron energy discrimination ratio, with $E_{\max }[5 \mathrm{MeV}] /$ $E_{\text {max }}[1 \mathrm{MeV}] \approx 3.5$ and 2.5 respectively.

The D-PC detector did provided the best gamma cross-sensitivity ratio $\left(E_{\max }[\mathrm{AmBe}] / E_{\max }[\mathrm{Co}-60] \approx 16\right)$ due to the low gamma interaction probability of the material $(Z)$. D-SC would be expected to perform similarly but the detector was 


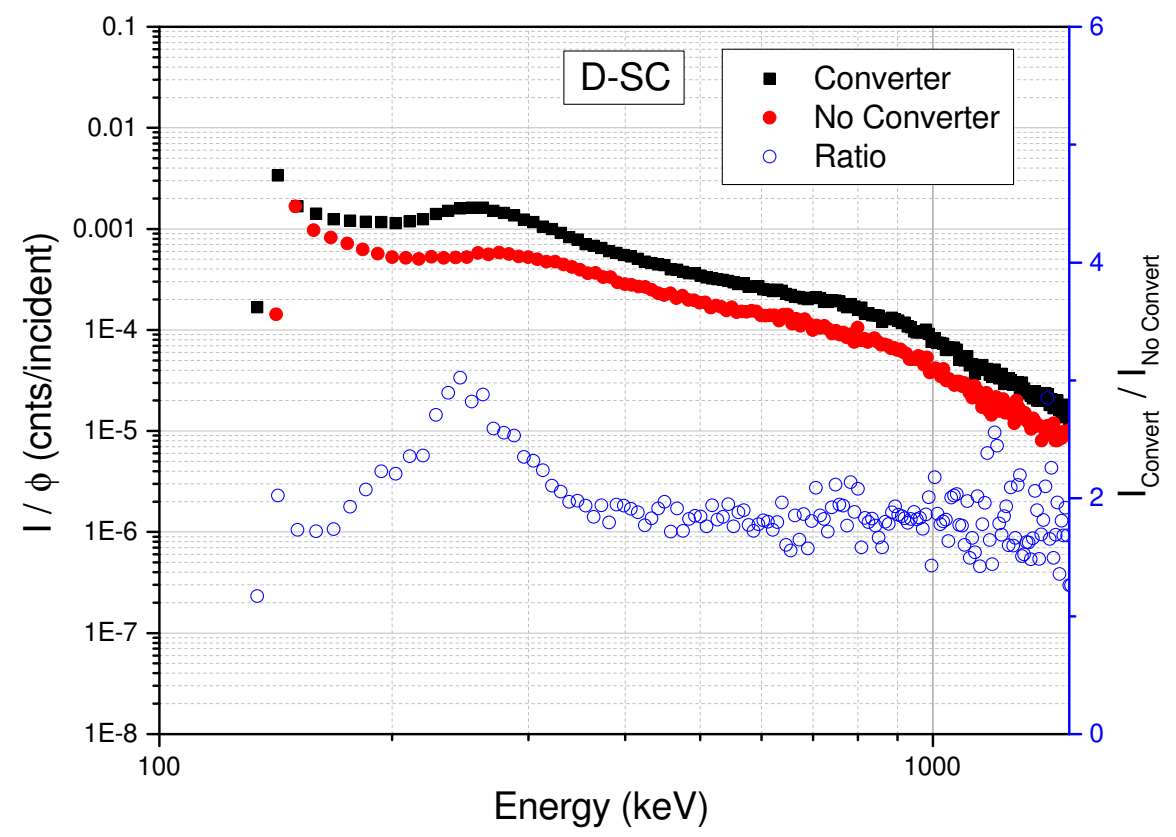

Figure 4. Count rate $(I)$, normalised to incident flux $(\phi)$, against energy $(E)$ for D-SC detector irradiated with AWE AmBe neutron source $(\approx 6 \mathrm{mSv} / \mathrm{h})$ at $-200 \mathrm{~V}$. Convertor is a $25 \mu \mathrm{m}$ Kapton layer. The ratio of counts per channel with $\left(I_{\text {Convert }}\right)$ and without $\left(I_{\text {No Convert }}\right)$ convertor layer has also been plotted as a function of energy.

thicker $(500 \mu \mathrm{m}$ compared to $300 \mu \mathrm{m})$ and as such had a lower neutron/gamma ratio (8.1) due to an increased gamma interaction probability. Similarly the thicker detector led to an observed 'peak' in the ${ }^{90} \mathrm{Sr} /{ }^{90} \mathrm{Y}$ beta spectrum (Figure 3) attributed to ${ }^{90} \mathrm{Sr}$ emissions $\left(E_{\max }[\mathrm{Sr}-90]=0.546 \mathrm{MeV}\right)$ when losses due to air are considered.

\section{Gamma and Neutron Intrinsic Efficiency}

Using the results presented in Table 1, the intrinsic efficiency has been normalised to the depletion width (calculated from capacitance measurements) in Figure 5 for gamma and neutron energies. This could effectively be regarded as the detection efficiency per unit of active volume for detector, a very important factor when considering the overall design, cost and eventual scaling for the applications discussed.

Taking the thickness of the detector region into consideration shows that the D-SC actually provides the lowest intrinsic efficiency for low energy ionising photons as per Figure 5 .

The $\mathrm{SiC}-\mathrm{EP}$ is quite insensitive to gamma radiation, as would be expected from the low $Z$ number and narrow depletion width in self-biased operation. Furthermore the calibration setup of the detector is such the noise edge (the point at which electronic noise in the system produces a non-negligible number of counts in the absence of a radiation source) was at least at $0.750 \mathrm{MeV}$ with a slightly higher noise edge used during the neutron testing $(\approx 1 \mathrm{MeV})$ due to increased noise in the system. Although an order of 


\begin{tabular}{|c|c|c|c|}
\cline { 2 - 4 } \multicolumn{1}{c|}{} & \multicolumn{3}{c|}{ Intrinsic } \\
Efficiency \\
\hline Detector & No Convertor & Convertor & Error \\
\hline D-SC & $2.59 \%$ & $5.35 \%$ & $\pm 0.01 \%$ \\
D-PC & $2.234 \%$ & - & $\pm 0.004 \%$ \\
SiC-EP & $0.010 \%$ & $0.011 \%$ & \pm 0.002 \\
SiC-SI & $0.42 \%$ & $1.26 \%$ & $\pm 0.04 \%$ \\
Si-PIN & $0.78 \%$ & $2.63 \%$ & $\pm 0.04 \%$ \\
\hline
\end{tabular}

Table 3. Calculated intrinsic efficiencies for detectors under irradiation of AmBe neutron source $(\approx 6 \mathrm{mSv} / \mathrm{h})$, with and without $25 \mu \mathrm{m}$ Kapton convertor layer.

magnitude larger than the gamma intrinsic efficiency, the neutron efficiency is also quite low in this detector, but this could feasibly be compensated for in large area applications with a combined array of detectors.

Similarly the SiC-SI detector demonstrated a lower overall gamma sensitivity relative to Si-PIN (Figure 5), which would be expected for the lower atomic number $(Z)$ of the material. However, both $\mathrm{SiC}-\mathrm{EP}$ and SiC-SI detectors actually demonstrated the lowest gamma cross-sensitivity ratios relative to Co-60 (6.4 and 6.9 respectively).

Although the Si-PIN direct neutron detection capability has been shown to be comparable, the reduced overall gamma sensitivity of the carbon based detectors ensures that lower energy thresholds can be used allowing for a more sensitive neutron detector. These results are of particular importance for Electronic Personal Neutron Dosimtery applications, where a reduced neutron-gamma cross-sensitivitiy leads to lower detector thresholds and potentially improved energy response.

It is worth noting that in Figure 5, the radionuclide sources (highlighted in the graphs) have been plotted using their average energy. For mono-energetic neutron sources the emission energy is very well defined, with some facilities capable of determining energies to within $\pm 1.5 \%$. For radionuclide sources however, there is a very wide range of neutron energies emitted due to the complex nature of the interactions occurring. With Cf-252 and AmBe radioisotope neutron sources, for example, despite having an average energy of $2.1 \mathrm{MeV}$ and $4.1 \mathrm{MeV}$, neutrons can be emitted with energies ranging up to $15 \mathrm{MeV}$ and $11 \mathrm{MeV}$ respectively [56], however for comparative purposes the average energies are included.

\section{Fast Neutron Convertor Layers}

The benefit of a hydrogenous converter layer has been demonstrated in Figure 4 and Table 3, with clear increases in the intrinsic efficiency for D-SC, SiC-SI and Si-PIN by factors of $2-3 \times$.

There was no significant improvement in the SiC-EP but this was to be expected due to the very thin depletion width when the applied bias is $0 \mathrm{~V}(1.3 \mu \mathrm{m}$ from Table 1$)$, 


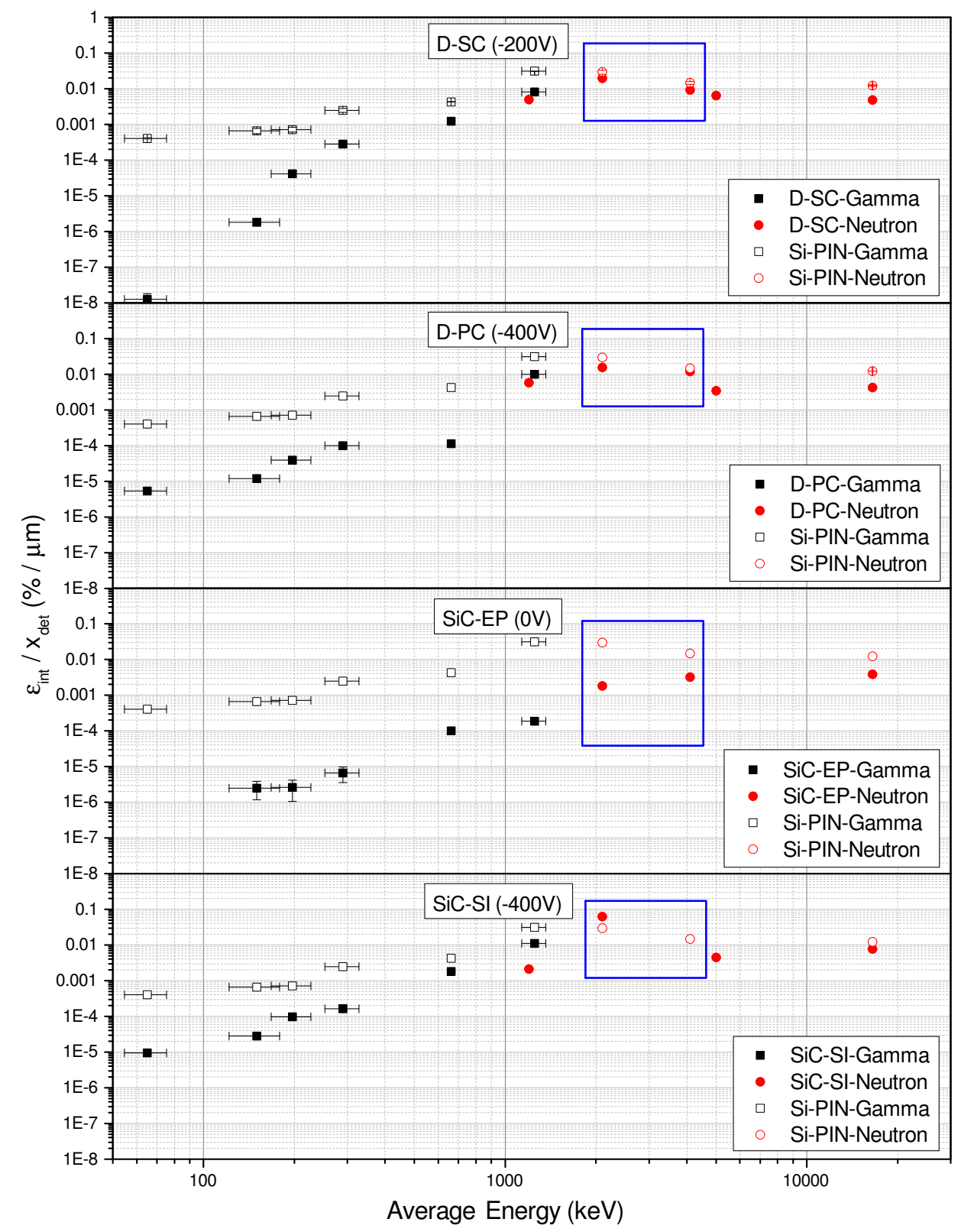

Figure 5. Depletion thickness compensated intrinsic efficiency $\left(\epsilon_{\text {int }} / x_{\text {det }}\right)$ against energy for X-rays, gammas and mono-energetic neutrons $(1.2 \mathrm{MeV}, 5.0 \mathrm{MeV}, 16.5 \mathrm{MeV})$. Highlighted area shows the average neutron energy emissions for the radionuclide sources $(\mathrm{Cf}-252=2.1 \mathrm{MeV}, \mathrm{AmBe}=4.1 \mathrm{MeV})$. All data were acquired at $\approx 6 \mathrm{mSv} / \mathrm{h}$.

as well as the large penetration depths of the conversion protons (18 $\mu \mathrm{m}$ from SRIM [53]). The application of the conversion layer was particularly difficult for the D-PC due to a large bonding wire and subsequent increased distance between the layer and detector, as such the measurement could not be made. 


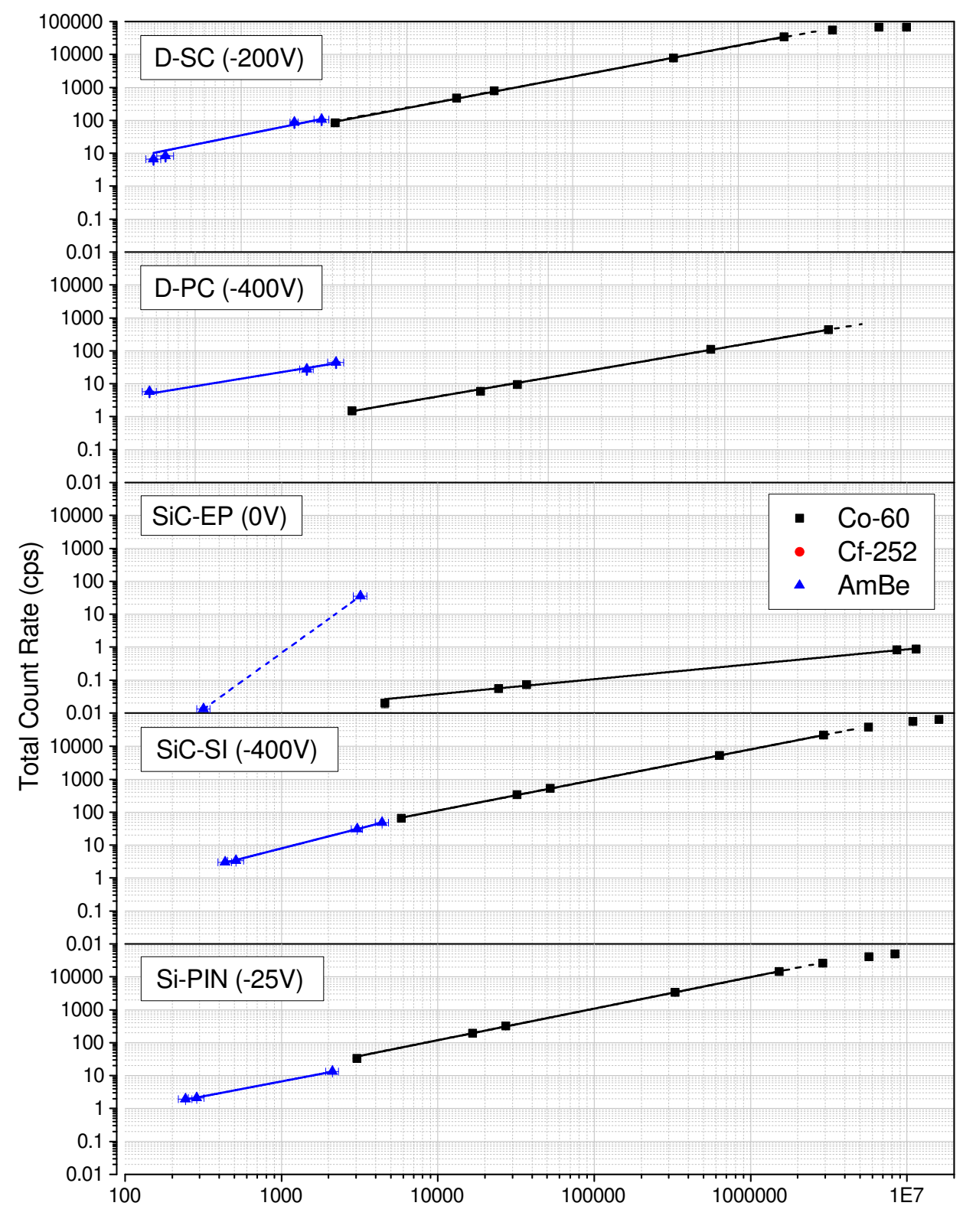

Flux (/s)

Figure 6. Total count rate above a given threshold against incident radiation flux for Co-60 gamma radiation and AmBe neutron radiation. The dashed line represents non-linear operation caused by pulse pile up in the multi channel analyser, for the $\mathrm{SiC}-\mathrm{EP}$ detector this was a result of noise alone. 


\section{Dose Rate Dependence}

As can be seen in Figure 6 the performance of these detectors is maintained over a wide dynamic range of neutron and gamma fluxes. This shows that the data are a result of direct gamma and neutron detection and not electronic noise or interference as the counting efficiencies are only dependent upon the radiation dose received. At very high fluxes the count rate becomes non-linear due to pulse pile up within the multi-channel analyser.

\section{Polarization Effects}

With the exception of the SiC-EP and Si-PIN, all the detectors demonstrated a change in the acquired spectrum and / or count rate with time, the so-called polarisation effect $[44][45]$.

This is a result of charge carriers being trapped in deep level locations for long periods of time, leading to a change in the space charge region and is very dependent upon the density of ionisation within the detectors, with radiation that results in a large ionisation concentrations in a small volume (such as alphas) producing very quick polarisation effects, as demonstrated in Figure 7. This is because these particles tend to create a large concentration of charge carriers at shallow depths within the detector $(\approx 17 \mu \mathrm{m}$ for diamond and $\approx 18 \mu \mathrm{m}$ for $\mathrm{SiC}[53])$ resulting in a high trapping rate over a small region [41][42][43]. This leads to the creation of a localised space charge barrier close to the electrode, through which further electrons and holes must pass to be fully collected.

A stable count rate was observed for the D-SC detector during beta, neutron and neutron+conversion proton irradiations, suggesting no polarisation. This is to be expected as these radiation types would result in less ionisation per incident radiation particle and therefore less trapping.

Furthermore a more uniform creation of electron hole pairs across the detector thickness would be expected for these radiation types, resulting in the trapped charges being distributed more evenly, diluting the overall space charge build-up and reducing the polarisation effect [57].

Interestingly for D-SC, the Co-60 gamma irradiation has been shown to actually increase the count rate and effectively enhance the detection capability, whereas alpha polarisation has been shown to recover over longer time periods. These observations demonstrate priming whereby the traps within the material are steadily filled with the created charge carriers until they reach saturation point, after which a stable field, and therefore spectrum, is maintained [42][57].

For the alpha particles the count rate initially decreases as initial charge carriers are trapped and incomplete charge collection in the detector occurs, resulting in some of the created pulse being less than the discriminator level. As the traps reach saturation,

further charge carriers are again able to fully traverse the detector, resulting in the count rate returning to normal and stabilising, demonstrating shallow priming. It should also 


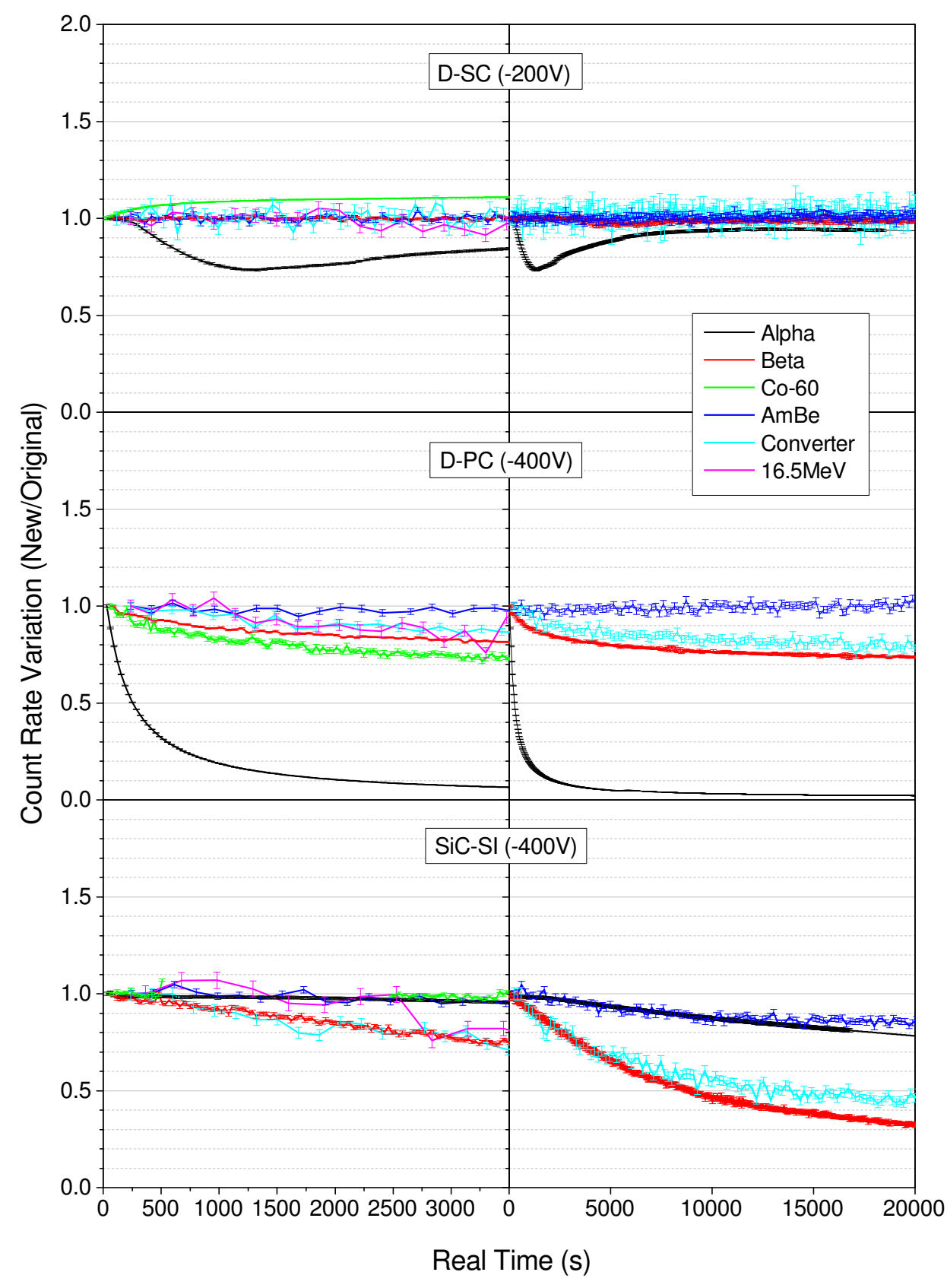

Figure 7. Count rate variation $\left(I / I_{0}\right)$ against time as a function of radiation type. Tests were conducted over 3600s and 20,000s. Alpha source was Am-241 (185kBq) in a $8 \times 10^{-2}$ mbar vacuum. AmBe was $\approx 18 \mathrm{mSv} / \mathrm{h}$, whereas the converter represents the AmBe neutron irradiation at $\approx 6 \mathrm{mSv} / \mathrm{h}$ with a $25 \mu \mathrm{m}$ Kapton proton conversion layer. Co-60 gammas and $16.5 \mathrm{MeV}$ neutron data was taken at $\approx 6 \mathrm{mSv} / \mathrm{h}$. 


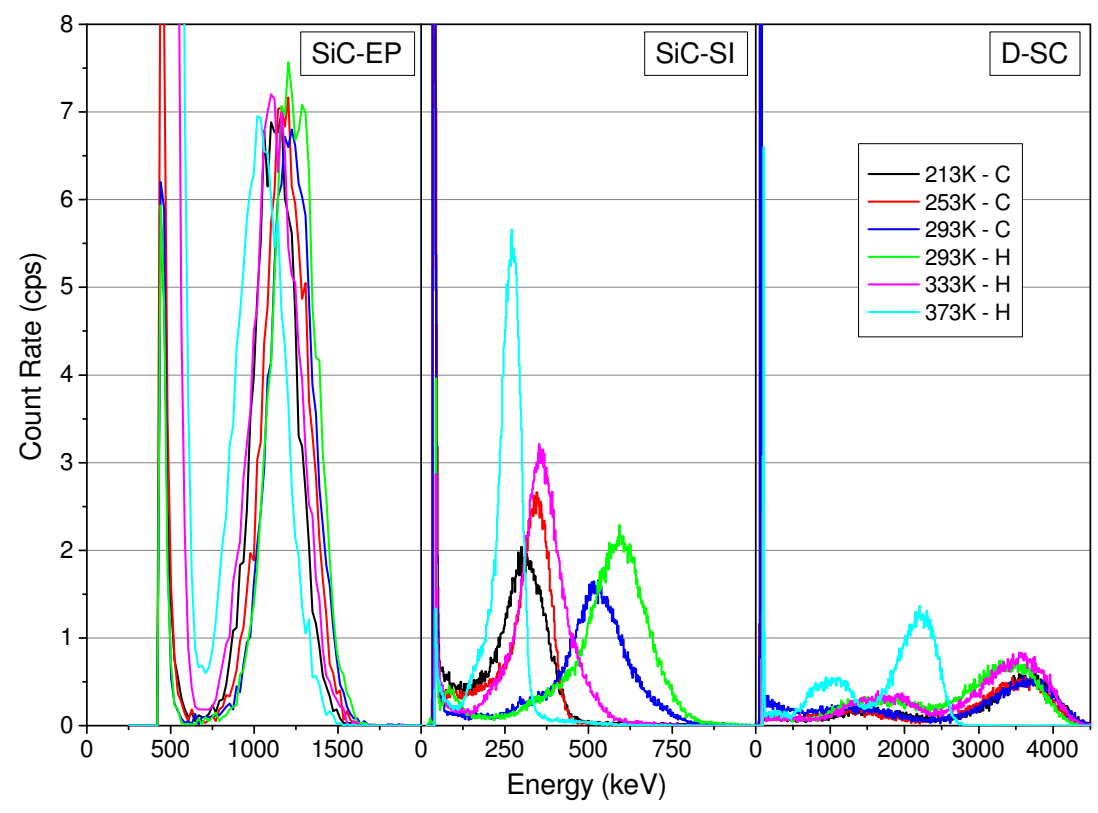

Figure 8. Count rate against energy as a function of temperature for an $60 \mathrm{kBq} \mathrm{Am}-$ 241 alpha radiation source. Suffix 'C' and ' $\mathrm{H}$ ' represent the cryostat used for cooling and heating respectively. Data was taken at $293 \mathrm{~K}$ in both cryostats for comparison. All testing was conducted at $6.4 \times 10^{-1}$ mbar.

be noted though that the trapped charges do create an additional space charge barrier, inhibiting the movement of further charge carriers and resulting in an observed reduced, but stable, alpha energy peak position.

The Co-60 irradiation on the other hand demonstrates uniform priming where initial charge carriers fill traps within the material leading to incomplete charge collection and an initial low count rate. However, future charge carriers can more easily traverse the material without being trapped and so the count rate will increase. As the trapped charge is evenly distributed throughout the detector there is not a localised space charge barrier for the charge carrier to overcome and there is little or no effect on the spectra position.

The quality of the D-SC material is such that the number of traps is significantly less than the rate of charge generation, essentially traps are rapidly filled and equilibrium is reached quickly. For D-PC and SiC-SI it is clear from Figure 7 that there are significantly more trap sites within the material as equilibrium is not reached and polarisation is observed for nearly all exposures.

As would be expected, the polarisation rate is very dependent upon the ionisation density of each radiation type and the distribution of the created charge carriers in the detector. In general, alpha particles show the greatest polarisation rate (high energy, full deposition of energy in material, highly concentrated charge carrier creation), followed by beta particles (lower energy, higher penetration) and then conversion protons (lower stopping power, higher penetration subject to the incident neutron energy). 
For alpha particles in particular, the polarisation tends towards debilitating effects within D-PC as the spectra move into the noise region and no further pulses can be registered. This is primarily due to the large emission rate of this source and the shallow penetration depth. This effect is not as apparent within the SiC-SI data, despite being demonstrated previously [27]. This is likely due to these exposures being conducted in air, resulting in a lower incident flux upon the detector which would be closer to the trapping/detrapping equilibrium.

Generally, polarisation (rather than priming) seems to dominate when charge carriers are not generated throughout the whole active thickness of the device.

\begin{tabular}{|c|c|c|c|c|c|}
\hline Detector & Bias & $\begin{array}{c}\text { Neutron } \\
\text { Discrimination } \\
\left(\frac{E_{\max }[n=5 \mathrm{MeV}]}{E_{\max }[n=1 \mathrm{MeV}]}\right)\end{array}$ & $\begin{array}{c}\text { Cross- } \\
\text { Sensitivity } \\
\left(\frac{E_{\max }[\mathrm{AmBe}]}{E_{\max }[\mathrm{Co}-60]}\right)\end{array}$ & $£ / \mathrm{mm}^{2}$ & $£ / \epsilon_{i}[\mathrm{AmBe}]$ \\
\hline D-SC & $-200 \mathrm{~V}$ & 3.5 & 8.1 & 98.76 & 900 \\
D-PC & $-400 \mathrm{~V}$ & 2.5 & 16.0 & 3.43 & 1334 \\
SiC-EP & 0V & $5.0^{*}$ & 6.4 & 3.40 & $3 \times 10^{6}$ \\
SiC-SI & $-400 \mathrm{~V}$ & 4.9 & 6.9 & 0.59 & 4206 \\
Si-PIN & $-25 \mathrm{~V}$ & $2.0^{*}$ & 9.1 & 0.02 & 284 \\
\hline
\end{tabular}

Table 4. Summary of key findings. The cost per unit efficiency is given by $£ / \epsilon_{i}[\mathrm{AmBe}]$ and was derived from wafer costs following conversations with suppliers, additional processing has not been included. ${ }^{*}$ Denotes neutron energy discrimination determined from the ratio of AmBe and Cf-252, $E_{\max }[A m B e] / E_{\max }[C f-252]$.

For the D-PC detector a stable count rate was observed for the the AmBe neutron exposure, despite the SiC-SI detector showing polarisation effect for the same exposure. As the D-PC detector had a larger area, it is possible that this is simply an effect of a reduced ionisation concentration over the whole detector (i.e. for the same dose rate, the flux at the boundary of the D-PC will be less than the SiC-SI). As such the reduced uniform trapping rate throughout the detector would be closer to equilibrium with the detrapping rate, resulting in no observable net polarisation effect. Certainly neutron polarisation has been observed in D-PC using a very high flux Cf-252 neutrons with the same principle [27].

It is also possible that this effect could be related to the enclosures the detectors were tested in, with the D-PC enclosure likely to produce more scattered electrons from Co-60 exposure (polarisation observed) and the SiC-SI enclosure likely to produce more recoil particles from neutron exposures (polarisation observed). These effects could be avoided in future testing by placing a low $Z$ filter (e.g. Al) between the source holders and detector to absorb the conversion particles, a common feature in radiation detection instrumentation.

It is worth noting that despite the onset of polarisation effects, with the potential exception of the alpha source exposures, operation was still possible for all detectors. 
For the diamond detectors in particular, the count rate rapidly stabilises following irradiation, demonstrating the point at which trapping and de-trapping is in equilibrium. As such all the detectors may be suitable for use in the applications discussed with suitable polarisation management techniques [43][58][59][60][61][62][63].

In fact, the selection of detectors for a particular application should be carefully considered. Diamond detectors are often considered to be quite expensive, as shown for the cost per unit area in Table 4. However, for direct neutron detection, the cost is actually comparable to the cheaper Si-PIN material when considering the relative efficiency. Furthermore, although Si-PIN detectors are not naturally thought of as direct neutron detectors the Si-PIN detector has been shown to be cheapest detector overall essentially they detect more neutrons per pound sterling than the alternatives tested.

\section{Temperature}

The effect of temperature on the alpha detection capability of SiC-EP, SiC-SI and D-SC has been shown in Figure 8. It was observed that the alpha detection characteristics of the D-SC detector are constant over all temperatures tested up until 373K, at which the peak position reduces by around around $60 \%$ and the full-width-half-maximum doubles.

For the SiC-SI detector there are variations in the alpha peak position $\left(P_{T}\right)$ with all temperatures tested, although the detector remains capable of detection at each point. It is also interesting to note that the maximum peak position $\left(P_{\max }\right)$ is achieved at room temperature $(293 \mathrm{~K})$. Altering the temperature by $\pm 40 \mathrm{~K}$ reduces the peak position by $\approx 40-45 \%$ and altering the temperature $\pm 80 \mathrm{~K}$ by decreases the peak by $\approx 40-55 \%$, suggesting a Gaussian style relationship of the order,

$$
\frac{P_{T}}{P_{\max }}=y_{0}+\left(\frac{A}{w \sqrt{\pi / 2}}\right) \cdot e^{-2\left(\left(T-x_{c}\right) / w\right)^{2}}
$$

where $T$ is the temperature. The values $y_{0}, A, w$ and $x_{c}$ are all constants derived from the Origin software using a Levenberg Marquardt empirical iteration function [][64], given by,

$$
\begin{aligned}
& c c l y_{0}=0.51 \pm 0.07 \\
& A \quad=287 \pm 9(K) \\
& w \quad=44 \pm 15(K) \\
& x_{c}=27 \pm 11
\end{aligned}
$$

but it is worth noting the $R^{2}$ fit is 0.77 due to non-symmetry at the extremes, as shown in Figure 9.

For the self-biased SiC-EP detector, there is little or no variation in the peak position or resolution over the range of temperature tested. Combined with the work presented by Abubakar et al. [4] which takes SiC-EP up to 500K, self-biased epitaxial $\mathrm{SiC}$ detectors seem very promising for harsh environment applications (i.e. reduced electronics, intrinsically safe operation). 


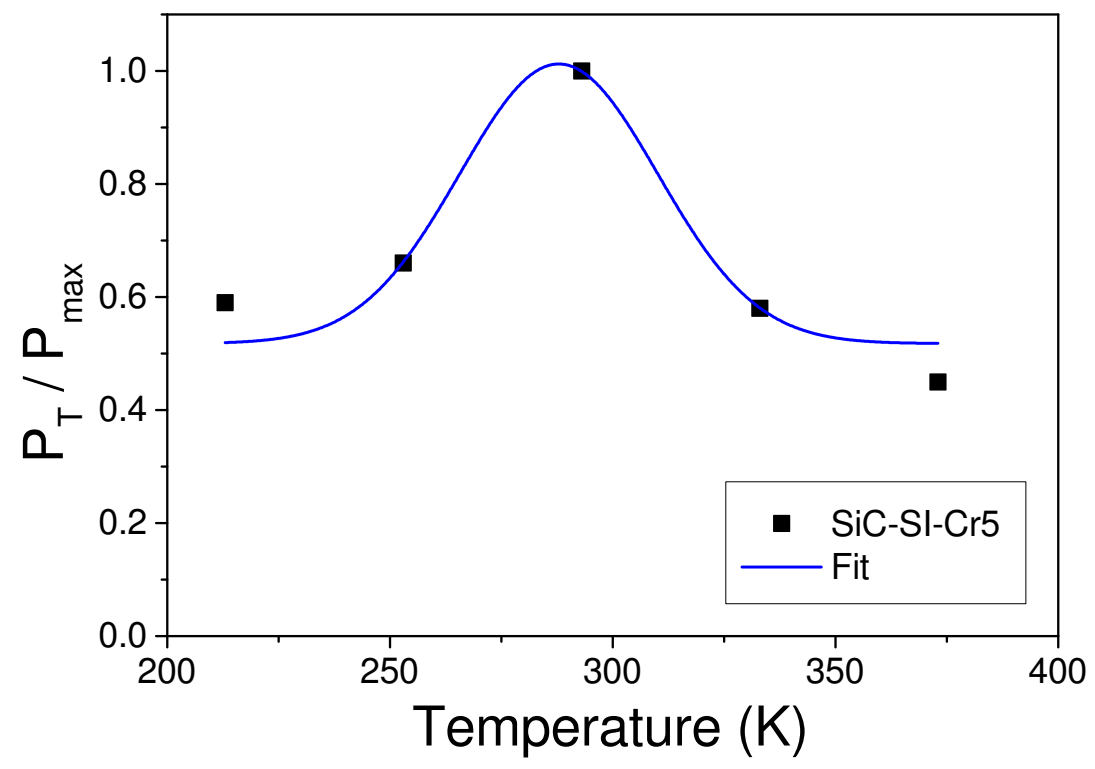

Figure 9. Variation of peak position $\left(P_{T}\right)$ relative to position at $293 \mathrm{~K}\left(P_{\max }\right)$ as a function of temperature for SiC-SI-Cr5 at $-400 \mathrm{~V}$ applied bias. Fit is a Origin Gaussian function using Levenberg Marquardt empirical iterations.

The leakage currents of the detectors while under bias increased with temperature as an exponential function $\left(\propto e^{\frac{-1}{T}}\right)$ but were still well within operational capabilities [36]. The leakage currents of the D-SC detector (at $-200 \mathrm{~V}$ ) varied from $I_{213 K} \approx 0.1 \mathrm{fA}$ to $I_{373 K} \approx 0.1 \mathrm{nA}$ and from $I_{213 K} \approx 1 \mathrm{fA}$ to $I_{373 K}<10 \mathrm{nA}$ for the SiC-SI-Cr5 (at $-400 \mathrm{~V}$ ) detector. No significant variation in leakage current was observed for the SiC-EP at $(0 \mathrm{~V})$, but variation was more pronounced when under bias negative bias $(\approx \mathrm{mA}$ at $40 \mathrm{~V})$.

\section{Conclusion}

A thorough investigation has been carried out in order to determine the suitability of diamond and silicon carbide detectors for specialist neutron detection applications such as Active Interrogation, Electronic Personal Neutron Dosimetry and Harsh Environments. A complete set of radiation exposures including alpha, beta, X-ray, gamma, radionuclide neutron and mono-energetic neutrons have demonstrated the detection capability of epitaxial $\mathrm{SiC}$ ( $\mathrm{SiC}-\mathrm{EP}$ ), semi-insulating $\mathrm{SiC}$ (SiC-SI), single crystal diamond (D-SC) and polycrystalline diamond (D-PC) over a wide dynamic range, with comparison against a commercial Si-PIN detector as a benchmark. Furthermore, the stability of the detectors while under radiation was shown, along with the performance of the SiC-EP, SiC-SI and D-SC in temperatures ranging from $-60^{\circ} \mathrm{C}$ to $+100^{\circ} \mathrm{C}$.

During the investigation stability issues were noted in the low doped, wide band gap materials (D-SC, D-PC and SiC-SI detectors), that being a change of both peak 
position and count rate over time, the so-called polarisation effect.

For D-SC, alpha radiation exposure caused an initial decrease in the count rate followed by a recovery to the initial rate, highlighting shallow priming, where by all the traps are filled at a shallow location within detector creating a space charge barrier for future charge carriers. For Co-60 gamma-ray irradiations the count rate actually increased initially, followed by stable operation, demonstrating uniform priming by which all the traps are filled uniformly throughout the detector diluting the space charge variation. For all other exposures the detector remained stable.

Polarisation was observed in the D-PC and SiC-SI detectors for alpha, beta, gamma, radionuclide neutron (fast and thermal), mono energetic neutron (fast) and neutron+proton (convertor) irradiations, with the exception of AmBe neutrons for DPC and Co-60 gamma-rays for SiC-SI. The results obtained generally showed an increase in polarisation rate as the ionisation density of the incident particle increased (i.e. the number of charge carriers created per incident particle). For D-PC this was likely down a lower overall incident flux relative to the other detectors as certainly radionuclide polarisation has been demonstrated for higher Cf-252 fluxes [27].

Despite this, with the exception of the alpha irradiations, the polarisation within the detectors was non-debilitating over the time period tested and as such stable operation was possible after the initial change in performance.

Consequently the detectors were shown to be able to directly detect and distinguish both the different radiation types and energies using a simple energy threshold discrimination method, with the $\mathrm{SiC}$ carbide detectors demonstrating the best neutron discrimination ratio and the Si-PIN demonstrating the lowest $\left(E_{\max }[\mathrm{n}=5 \mathrm{MeV}] /\right.$ $E_{\text {max }}[\mathrm{n}=1 \mathrm{MeV}] \approx 5$ and 2 respectively). Essentially this ratio suggests it is easier to discriminate between the two neutron energies in $\mathrm{SiC}$ than in $\mathrm{Si}$.

However, the $\mathrm{SiC}$ detectors did demonstrate the lowest neutron-gamma crosssensitivity ratio relative to Co-60 photons $\left(E_{\max }[\mathrm{AmBe}] / E_{\max }[\mathrm{Co}-60] \approx 6.5\right)$, with D$\mathrm{PC}$ providing the best ratio (16). This suggests that there could be more difficulty in discriminating gamma-ray interference from direct neutron detection in the $\mathrm{SiC}$ relative to the other detectors. Further work was presented showing that these cross-sensitivity ratios can be improved through use of a simple proton-recoil conversion layer and that the intrinsic gamma efficiency of the carbon based detectors was less than the Si detector for lower energy ionising photons.

The work conducted also showed that SiC-EP could be used as a effective neutron detector when operated under self-bias. Neutron and alpha radiation could be clearly discriminated, where as the ionising photon intrinsic efficiency was shown to be significantly lower than the other detectors due to the low $\mathrm{Z}$ number, narrow depletion width and calibration setup (i.e. low minimum energy threshold, $<0.750 \mathrm{kMeV}$ ).

Finally work was conducted to demonstrate that SiC-EP, semi-insulating $\mathrm{SiC}$ and $\mathrm{D}-\mathrm{SC}$ detectors could be operated in a wide range of environmental temperatures. Minor variations in alpha energy peak position were noticed for the the $\mathrm{SiC}-\mathrm{Si}$ and $\mathrm{D}-\mathrm{SC}$ detectors although detection capability was still maintained over $-60^{\circ} \mathrm{C}$ to $+100^{\circ} \mathrm{C}$. 
The self-biased SiC-EP detector on the other hand demonstrated no variation in alpha detection performance or quality for the range of temperatures tested.

\section{Acknowledgements}

The authors would like to acknowledge the staff at the University of Surrey, NPL`, Thermo Fisher Scientific ${ }^{\circledR}$ and AWE for there assistance with testing and analysis. The authors would also like to thank Loadpoint $\left.{ }^{(}\right)$for their work during the detector fabrication process.

This work was funded by the United Kingdom Science Technology Funding Council (STFC grant number: ST/H003959/1) and in collaboration with AWE. 
[1] Ruddy FH, Dulloo AR, Seidel JG, Das MK, Ryu SH, Agarwal AK. The fast neutron response of $4 \mathrm{H}$ silicon carbide semiconductor radiation detectors. Nuclear Science, IEEE Transactions on. 2006;53(3):1666-1670.

[2] Pillon M, Angelone M, Krása A, Plompen AJM, Schillebeeckx P, Sergi ML. Experimental response functions of a single-crystal diamond detector for $5-20.5 \mathrm{MeV}$ neutrons. Nuclear Instruments and Methods in Physics Research Section A: Accelerators, Spectrometers, Detectors and Associated Equipment. 2011 Jun;640(1):185-191.

[3] Wrbanek JD, Wrbanek SY, Fralick GC, Chen LY. Micro-fabricated solid-state radiation detectors for active personal dosimetry. NASA/TM. 2007;214674.

[4] Abubakar YM, Lohstroh A, Sellin PJ. Stability of Silicon Carbide Particle Detector Performance at Elevated Temperatures. IEEE Transactions on Nuclear Science. 2015 Oct;62(5):2360-2366.

[5] McGregor DS, Hammig MD, Yang YH, Gersch HK, Klann RT. Design considerations for thin film coated semiconductor thermal neutron detectors-I: basics regarding alpha particle emitting neutron reactive films. Nuclear Instruments and Methods in Physics Research Section A: Accelerators, Spectrometers, Detectors and Associated Equipment. 2003 Mar;500(1-3):272-308.

[6] Nava F, Bertuccio G, Cavallini A, Vittone E. Silicon carbide and its use as a radiation detector material. Measurement Science and Technology. 2008;19:102001.

[7] Sellin PJ, Vaitkus J. New materials for radiation hard semiconductor dectectors. Nuclear Instruments and Methods in Physics Research Section A: Accelerators, Spectrometers, Detectors and Associated Equipment. 2006 Feb;557(2):479-489.

[8] Angelone M, Fonnesu N, Pillon M, Prestopino G, Sarto F, Milani E, et al. Spectrometric performances of monocrystalline artificial diamond detectors operated at high temperature. IEEE; 2011. p. 2188-2191.

[9] Stevenson J, Gozani T, Elsalim M, Condron C, Brown C. Linac based photofission inspection system employing novel detection concepts. Nuclear Instruments and Methods in Physics Research Section A: Accelerators, Spectrometers, Detectors and Associated Equipment. 2011 Oct;652(1):124-128.

[10] Runkle RC, Chichester DL, Thompson SJ. Rattling nucleons: New developments in active interrogation of special nuclear material. Nuclear Instruments and Methods in Physics Research Section A: Accelerators, Spectrometers, Detectors and Associated Equipment. 2011;663:75-95.

[11] Gozani T. Fission Signatures for Nuclear Material Detection. IEEE Transactions on Nuclear Science. 2009 Jun;56(3):736 -741.

[12] Active Interrogation Project Requirements. Project Collaboration; 2012. Internal Report.

[13] Ginjaume M, Bolognese-Milsztajn T, Luszik-Bhadra M, Vanhavere F, Wahl W, Weeks A. Overview of active personal dosemeters for individual monitoring in the European Union. Radiation Protection Dosimetry. 2006 Dec;125(1-4):261-266.

[14] Chau Q, Lahaye T. Evaluation of dose equivalent by the electronic personal dosemeter for neutron 'Saphydose-N' at different workplaces of nuclear facilities. Radiation Protection Dosimetry. 2006 Dec;125(1-4):271-274.

[15] Luszik-Bhadra M, Matzke M, Dietz E, Guldbakke S, Hecker O, Sosaat W, et al. An active personal dosemeter/spectrometer for neutrons. Radiat Prot Dosim. 1999;84(1-4):375-380. WOS:000081587500086.

[16] Luszik-Bhadra M, Rosenfeld A, Kron T, d'Errico F, Moscovitch M. State of the Art in Electronic Dosemeters for Neutrons. AIP Conf. Proc. 1345; 2011. p. 262-273.

[17] d'Errico F, Luszik-Bhadra M, Lahaye T. State of the art of electronic personal dosimeters for neutrons. Nuclear Instruments and Methods in Physics Research Section A: Accelerators, Spectrometers, Detectors and Associated Equipment. 2003 Jun;505(1-2):411-414.

[18] Glasstone S, Sesonske A. Nuclear reactor engineering. 4th ed. New York, NY: Chapman \& Hall; 1994.

[19] Steele LE. Neutron irradiation embrittlement of reactor pressure vessel steels. Technical reports series - International Atomic Energy Agency ; no. 163. Vienna: International Atomic Energy 
Agency; 1975.

[20] ANALYSIS AND UPGRADE OF INSTRUMENTATION AND CONTROL SYSTEMS FOR THE MODERNIZATION OF RESEARCH REACTORS. IAEA; 1990. TECDOC-540. Available from: http://www.iaea.org/inis/collection/NCLCollectionStore/_Public/ 19/068/19068608.pdf.

[21] STEP 3 STRUCTURAL INTEGRITY ASSESSMENT OF THE EDF and AREVA UK EPR DIVISION 6 ASSESSMENT REPORT NO. AR 09/012-P. HSE Nuclear Directorate;. AR 09/012-P.

[22] Luszik-Bhadra M, Matzke M, Schuhmacher H. Development of personal neutron dosemeters at the PTB and first measurements in the space station MIR. Radiation Measurements. 2001 Jun;33(3):305-312.

[23] Durante M. Space radiation protection: Destination Mars. Life Sciences in Space Research. 2014 Apr;1:2-9. Available from: http://linkinghub.elsevier.com/retrieve/ $\mathrm{pii/S2214552414000042.}$

[24] Werner MR, Fahrner WR. Review on materials, microsensors, systems and devices for hightemperature and harsh-environment applications. IEEE Transactions on Industrial Electronics. 2001;48(2):249-257.

[25] Nikitin A, Bliven S. Needs of well logging industry in new nuclear detectors. In: IEEE Nuclear Science Symposuim \& Medical Imaging Conference. IEEE; 2010. p. 1214-1219.

[26] Abdel-Rahman MAE, Lohstroh A, Sellin PJ. The effect of annealing on the X-ray induced photocurrent characteristics of CVD diamond radiation detectors with different electrical contacts. Physica Status Solidi a-Applications and Materials Science. 2011;208(9):2079-2086.

[27] Hodgson M, Lohstroh A, Sellin P, Thomas D. Neutron detection performance of silicon carbide and diamond detectors with incomplete charge collection properties. Nuclear Instruments and Methods in Physics Research Section A: Accelerators, Spectrometers, Detectors and Associated Equipment. 2017 Mar;847:1-9.

[28] Schirru F, Singh BSN, Scruton L, Bentley MA, Fox SP, Lohstroh A, et al. Development of large area polycrystalline diamond detectors for fast timing application of high-energy heavyion beams. Journal of Instrumentation. 2012 May;7(05):P05005-P05005.

[29] Bruzzi M, Miglio S, Tosi C, Manfredotti C, LoGiudice A, Vittone E, et al.. RD50 - The SiC Common Structures Project. CERN;.

[30] Bruzzi M, Lagomarsino S, Nava F, Sciortino S. Characterisation of epitaxial SiC Schottky barriers as particle detectors. Diamond and related materials. 2003;12(3):1205-1208.

[31] Bertuccio G, Caccia S, Puglisi D, Macera D. Advances in silicon carbide X-ray detectors. Nuclear Instruments and Methods in Physics Research Section A: Accelerators, Spectrometers, Detectors and Associated Equipment. 2011 Oct;652(1):193-196.

[32] Bruzzi M, Nava F, Russo S, Sciortino S, Vanni P. Characterisation of silicon carbide detectors response to electron and photon irradiation. Diamond and related materials. 2001;10(3):657661.

[33] Ruddy FH, Seidel JG, Flammang RW, Singh R, Schroeder J. Development of radiation detectors based on semi-insulating silicon carbide. In: IEEE Nuclear Science Symposium Conference Record. NSS '08; 2008. p. $449-455$.

[34] Nava F, Vanni P, Bruzzi M, Lagomarsino S, Sciortino S, Wagner G, et al. Minimum Ionizing and Alpha Particles Detectors Based on Epitaxial Semiconductor Silicon Carbide. IEEE Transactions on Nuclear Science. 2004 Feb;51(1):238-244.

[35] Ha JH, Kang SM, Park SH, Kim HS, Lee NH, Song TY. A self-biased neutron detector based on an $\mathrm{SiC}$ semiconductor for a harsh environment. Applied Radiation and Isotopes. $2009 \mathrm{Jul} ; 67$ (78):1204-1207.

[36] Knoll GF. Radiation detection and measurement. 4th ed. Hoboken, N.J: John Wiley; 2010. ISBN 97804701314800470131489.

[37] Gooda PH, Gilboy WB. High resolution alpha spectroscopy with low cost photodiodes. Nuclear 
Instruments and Methods in Physics Research Section A: Accelerators, Spectrometers, Detectors and Associated Equipment. 1987;255(1):222-224.

[38] Bolotnikov AE, Boggs SE, Hubert Chen CM, Cook WR, Harrison FA, Schindler SM. Properties of Pt Schottky type contacts on high-resistivity CdZnTe detectors. Nuclear Instruments and Methods in Physics Research Section A: Accelerators, Spectrometers, Detectors and Associated Equipment. 2002;482(1):395-407.

[39] Bolotnikov AE, Camarda GC, Wright GW, James RB. Factors limiting the performance of CdZnTe detectors. IEEE Transactions on Nuclear Science. 2005 Jun;52(3):589-598.

[40] Siegbahn K, editor. Alpha-, beta- and gamma-ray spectroscopy. Amsterdam: North-Holland Publ. [u.a.]; 1965. ISBN 0444106952.

[41] Rogalla M, Runge K, Söldner-Rembold A. Particle detectors based on semi-insulating silicon carbide. Nuclear Physics B-Proceedings Supplements. 1999;78(1):516-520.

[42] Lan L, Xiaoping O, Xinjian T, Liangbin X, Na C, Bing L, et al. Priming effect on a polycrystalline CVD diamond detector under 60-Co $\gamma$-rays irradiation. Nuclear Instruments and Methods in Physics Research Section A: Accelerators, Spectrometers, Detectors and Associated Equipment. 2012 Apr;672:29-32.

[43] Lohstroh A, Sellin PJ, Wang SG, Davies AW, Parkin JM. Mapping of polarization and detrapping effects in synthetic single crystal chemical vapor deposited diamond by ion beam induced charge imaging. Journal of Applied Physics. 2007;101(6):063711.

[44] Chynoweth AG. Behavior of Space Charge in Diamond Crystal Counters under Illumination. I. Physical Review. 1951;83(2):254.

[45] Ivanov AM, Lebedev AA, Strokan NB. Effect of extreme radiation fluences on parameters of SiC nuclear particle detectors. Semiconductors. 2006 Oct;40(10):1227-1231.

[46] Camarda GS, Bolotnikov AE, Cui Y, Hossain A, Awadalla SA, Mackenzie J, et al. Polarization Studies of CdZnTe Detectors Using Synchrotron X-Ray Radiation. IEEE Transactions on Nuclear Science. 2008 Dec;55(6):3725-3730.

[47] Siffert P, Berger J, Scharager C, Cornet A, Stuck R, Bell R, et al. Polarization in cadmium telluride nuclear radiation detectors. Nuclear Science, IEEE Transactions on. 1976;23(1):159-170.

[48] NCE/2. BS ISO 4037-3:1999 - X and gamma reference radiation for calibrating dosemeters and doserate meters and for determining their response as a function of photon energy. Calibration of area and personal dosemeters and the measurement of their response as a function of energy and angle of incidence. Roentgen- und Gamma-Referenzstrahlungsfelder fuer die Kalibrierung von Dosis- und Dosisleistungsmessgeraeten und fuer die Ermittlung des Ansprechvermoegens in Abhaengigkeit von der Photonenenergie. Kalibrierung von Orts- und Personendosimetern und Messung ihres Ansprechvermoegens in Abhaengigkeit von Energie und Einfallswinkel. International Organization for Standardization; 1999.

[49] Jones J, Blackburn B, Ruddy F, Seidel J, Flammang R. Novel Silicon Carbide Detector for Active Inspections. In: Government Microcircuit Applications and Critical Technology Conference. Orlando, USA: Idaho National Laboratory; 2007. .

[50] Zat'ko B, Sedlačková K, Dubecký F, Boháček P, Sekáčová M, Nečas V. Detection of fast neutrons using detectors based on semi-insulating GaAs. Journal of Instrumentation. 2011 Dec;6(12):C12047-C12047.

[51] Sasaki M, Nakamura T, Tsujimura N, Ueda O, Suzuki T. Development and characterization of real-time personal neutron dosemeter with two silicon detectors. Nuclear Instruments and Methods in Physics Research Section A: Accelerators, Spectrometers, Detectors and Associated Equipment. 1998;418(2):465-475.

[52] Andersson Sundén E, Sjöstrand H, Conroy S, Ericsson G, Gatu Johnson M, Giacomelli L, et al. The thin-foil magnetic proton recoil neutron spectrometer MPRu at JET. Nuclear Instruments and Methods in Physics Research Section A: Accelerators, Spectrometers, Detectors and Associated Equipment. 2009 Nov;610(3):682-699. Available from: http://linkinghub.elsevier.com/ retrieve/pii/S0168900209017483. 
[53] Ziegler J. SRIM \& TRIM; 2013. Accessed 07-07-2015.

[54] Ruby L. Further comments on the geometrical efficiency of a parallel-disk source and detector system. Nuclear Instruments and Methods in Physics Research Section A: Accelerators, Spectrometers, Detectors and Associated Equipment. 1994 Jan;337(2-3):531-533. Available from: http://linkinghub.elsevier.com/retrieve/pii/016890029491124X.

[55] Chichester D, Seabury E. Active Neutron Interrogation to Detect Shielded Fissionable Material. In: Proc. Int. Topical Meeting on Nuclear Research Applications and Utilization of Accelerators, International Atomic Energy Agency, Vienna, Austria; 2009. .

[56] NCE/2. BS ISO 8529-1:2001 - Reference neutron radiations. Characteristics and methods of production. Neutronen-Referenzstrahlungen. Merkmale und Verfahren zur Erzeugung. International Organization for Standardization; 2001.

[57] Souw EK, Meilunas RJ. Response of CVD diamond detectors to alpha radiation. Nuclear Instruments and Methods in Physics Research Section A: Accelerators, Spectrometers, Detectors and Associated Equipment. 1997 Nov;400(1):69-86.

[58] Malm H, Martini M. Polarization phenomena in CdTe nuclear radiation detectors. Nuclear Science, IEEE Transactions on. 1974;21(1):322-330.

[59] Mersi S, Borchi E, Bruzzi M, D'Alessandro R, Lagomarsino S, Sciortino S. A study of charge collection processes on polycrystalline diamond detectors. Nuclear Instruments and Methods in Physics Research Section A: Accelerators, Spectrometers, Detectors and Associated Equipment. 2004 Sep;530(1-2):146-151.

[60] Ivanov AM, Strokan NB, Lebedev AA. Radiation resistance of wide-gap materials as exemplified by SiC nuclear radiation detectors. Technical Physics. 2012 Apr;57(4):556-560.

[61] Bell R, Entine G, Serreze H. Time-Dependent Polarization of CdTe Gamma-Ray Detectors. Nuclear Instruments \& Methods. 1974;117(1):267-271.

[62] Giacomelli L, Andreani C, Fazzi A, Frost CD, Gorini G, Perelli Cippo E, et al. Diamond detectors for fast neutron irradiation experiments. Nuclear Physics B - Proceedings Supplements. 2011 Jun;215(1):242-246. Available from: http://linkinghub.elsevier.com/retrieve/pii/ S0920563211002593.

[63] Muraro A, Giacomelli L, Nocente M, Rebai M, Rigamonti D, Belli F, et al. First neutron spectroscopy measurements with a pixelated diamond detector at JET. Review of Scientific Instruments. 2016 Nov;87(11):11D833. Available from: http://scitation.aip.org/content/ aip/journal/rsi/87/11/10.1063/1.4961557.

[64] R L Buden, J D Faires. Numerical Analysis. 5th ed. PWS Publishing Company, Boston; 1993. 